\title{
Protecting a quantum state from environmental noise by an incompatible finite-time measurement
}

\author{
Carlos Alexandre Brasil $*$ L. A. de Castro $t$ and R. d. J. Napolitano \\ Instituto de Física de São Carlos, Universidade de São Paulo, \\ P.O. Box 369, 13560-970, São Carlos, SP, Brazil
}

\begin{abstract}
We show that measurements of finite duration performed on an open two-state system can protect the initial state from a phase-noisy environment, provided the measured observable does not commute with the perturbing interaction. When the measured observable commutes with the environmental interaction, the finite-duration measurement accelerates the rate of decoherence induced by the phase noise. For the description of the measurement of an observable that is incompatible with the interaction between system and environment, we have found an approximate analytical expression, valid at zero temperature and weak coupling with the measuring device. We have tested the validity of the analytical predictions against an exact numerical approach, based on the superoperator-splitting method, that confirms the protection of the initial state of the system. When the coupling between the system and the measuring apparatus increases beyond the range of validity of the analytical approximation, the initial state is still protected by the finite-time measurement, according with the exact numerical calculations.
\end{abstract}

PACS numbers: 03.65.-w Quantum mechanics, 03.65.Ta Foundations of quantum mechanics; measurement theory, 02.60.-x Numerical approximation and analysis

*Electronic address: carlosbrasil@ifsc.usp.br

${ }^{\dagger}$ Electronic address: leonardo.castro@usp.br 


\section{INTRODUCTION}

The problem of measurement is fundamental to quantum theory [1 5$]$. According to the standard interpretation [1, 3, 4], established after the pioneer contributions by Born [3, 6, 6], Dirac [8], and von Neumann [3], the postulated collapse of the wave function implies an instantaneous measurement process. However, the de facto meaning of a quantum measurement is the result of a physical interaction between the system being measured and the measuring apparatus. Such an interaction can be described by a suitable formalism [9, 10], which, under the hypothesis that the measurement process is irreversible, leads to the Lindblad equation [11 13]. This equation describes the evolution of a measurement during a finite time interval by the stochastic term - Lindbladian. At last, the information of the probabilities associated to every possible result is acquired by the conventional way with the density operator diagonal elements - populations [14, 15].

Based on superoperator algebra and Nakajima-Zwanzig projectors [16, 17], we have been able to describe the dynamics of an arbitrary measurement that occurs during a finite time interval, while the system being measured interacts with the rest of the universe and, due to the consequent environmentally-induced noise, undergoes decoherence [18]. The assumption that the interaction of the measuring apparatus with the system is Markovian justifies a Lindbladian approach. However, to treat the noise introduced by the fact that, during the finite-duration measurement, the system is perturbed by the environment, a Markovian approximation is too restrictive, since non-Markovian noise effects can become non-negligible for time scales in which coarse graining is not a good approximation [19 24]. Instead, to develop a formalism able to include non-Markovian effects, we have used a Redfield approach to the dynamical description of the interaction between the system and its environment, excluding the apparatus. After tracing out the degrees of freedom introduced to describe the non-Markovian noise, the resulting master equation in the Born approximation (not a BornMarkov approximation), referred only to the system, can be used to investigate the effects of the environmental noise on the measurement dynamics. We do not use additional Lindblad channels to describe the environmental noise because such a formulation would preclude any attempt to treat non-Markovian effects. Since we are interested in describing the effects of the noise during the finite-time measurement, turning off the environmental perturbation during the action of the measuring apparatus is not a valid approximation. Therefore, our 
hybrid approach is the most economical dynamical description of a measurement that is simultaneous to non-Markovian environmental perturbations.

The Lindblad equation can be used to describe Markovian and non-Markovian environmental effects, with applications ranging from decoherence and dissipation analyses [12, 15] to measurement processes [9], particularly when we consider that the measurements have finite durations [14, 15]. It follows as a mathematical consequence of a semi-group dynamics to describe irreversibility in quantum mechanics [11]. Therefore, it can only be used to describe Markovian processes, unless the number of dynamical variables describing the system be enlarged [20], causing computational overhead that we must avoid. Incidentally, we point out that the extension of the Lindblad equation to non-Markovian systems [20] can be applied to non-semigroup dynamics as well, with time-dependent Hamiltonian and Lindbladian operators.

In the present paper, we analyze finite-time measurements that commute or do not commute with the interaction Hamiltonian. We study a two-state system interacting with a bath of harmonic oscillators, that emulates a phase-noisy environment. The approximate analytical solution agrees with the numerical results of the superoperator-splitting method [25] for weak coupling between the system and its environment. We find that the finitetime measurement can protect the measured state, if the observable being measured does not commute with the Hamiltonian describing the noisy environmental interaction with the system but, when the measurement commutes with the interaction, the environment only increases the coherence decay. For strong coupling between the system and the environment, our analytical solution fails, as shown by a thorough comparison of its predictions against the corresponding exact numerical results. In the case of strong coupling, the effect predicted by the numerical calculations is a more intense error as the coupling with environment increases.

Subsequent researchers may use the method presented here to study the effects of varying the temperature, the density of states, or even the system considered, increasing the number of quantum states. There are some interesting possible directions to follow to extend the present investigation, such as the study of non-Markovian systems [19 24], time-dependent Nakajima-Zwanzig projectors [26, 27], higher-order approximations for the interaction between the system and its environment [28, 29], and effects of other kinds of environmental noise. One problem which the present theory can not address is the possibility that the 
measurement result be different from one of the eigenvalues of the meter [30].

The paper is structured as follows: In Sec. II we briefly present the proposed formalism and state the problem to be analyzed. In Sec. III, we present the approximate analytical solution to the master equation, followed by a derivation of its numerical counterpart in Sec. IV. In Sec. V, we discuss the results obtained with the expressions in Sec. III and Sec. IV and its physical implications. In Sec. VI, we present some perspectives for expanding the material that we have presented here and a conclusion.

\section{THE HYBRID MASTER EQUATION}

The Lindblad equation,

$$
\frac{d}{d t} \hat{\rho}_{S B}=-\frac{i}{\hbar}\left[\hat{H}, \hat{\rho}_{S B}\right]+\sum_{j}\left(\hat{L}_{j}^{(S)} \hat{\rho}_{S B} \hat{L}_{j}^{(S) \dagger}-\frac{1}{2}\left\{\hat{L}_{j}^{(S) \dagger} \hat{L}_{j}^{(S)}, \hat{\rho}_{S B}\right\}\right)
$$

where $\hat{\rho}_{S B}$ is the density operator describing the system and its environment, $\hat{H}$ is the total Hamiltonian and the $\hat{L}_{j}^{(S)}$ are the Lindblad operators, which act only on the system, is the most general form for a master equation [11, 13]. The second term on the right-hand side of Eq. (11),

$$
\sum_{j}\left(\hat{L}_{j}^{(S)} \hat{\rho}_{S B} \hat{L}_{j}^{(S) \dagger}-\frac{1}{2}\left\{\hat{L}_{j}^{(S) \dagger} \hat{L}_{j}^{(S)}, \hat{\rho}_{S B}\right\}\right),
$$

is the Lindbladian operator acting on the density operator $\hat{\rho}_{S B}$. The Liouvillian operator acting on $\hat{\rho}_{S B}$,

$$
-\frac{i}{\hbar}\left[\hat{H}, \hat{\rho}_{S B}\right]
$$

accounts for the unitary portion of the propagation, before the environmental degrees of freedom are traced out, and the Lindbladian represents the Markovian measurement dynamics.

We begin with a system $S$ and its environment $B$, whose interaction is described by the Hamiltonian:

$$
\hat{H}_{S B}=\sum_{k} \hat{S}_{k} \hat{B}_{k}
$$

where, for each index $k, \hat{S}_{k}$ operates only on the system $S$ and $\hat{B}_{k}$, only on the environment B. The form of the interaction, Eq. (2), is general enough, assumed by both amplitude 
and phase damping models [15]. Here, to account for non-Markovian noise, we choose to treat the environmental interaction as part of the total Hamiltonian, $\hat{H}$, appearing in the Liouvillian term of Eq. (11). Thus, we write

$$
\hat{H}=\hat{H}_{B}+\hat{H}_{S B}+\hat{H}_{S}
$$

where $\hat{H}_{B}$ is the environmental Hamiltonian and $\hat{H}_{S}$ is the system Hamiltonian. All the information read by the measuring apparatus, which is assumed to be Markovian, will be accounted for by the Lindbladian term of Eq. (11) and, as usual, the Lindblads $\hat{L}_{j}^{(S)}$ will act only on the Hilbert space of the system, since we are interested in measuring system observables only. Our aim is to obtain an equation for the time evolution of the reduced density matrix of the system, $\hat{\rho}_{S}$, that is,

$$
\hat{\rho}_{S}=\operatorname{Tr}_{B}\left\{\hat{\rho}_{S B}\right\}
$$

For any density matrix operator $\hat{X}$, let the superoperators $\hat{\hat{B}}, \hat{\hat{S}}$, and $\hat{\hat{F}}$ be defined, respectively, as

$$
\begin{gathered}
\hat{\hat{B}} \hat{X}=-\frac{i}{\hbar}\left[\hat{H}_{B}, \hat{X}\right] \\
\hat{\hat{S}} \hat{X}=-\frac{i}{\hbar}\left[\hat{H}_{S}, \hat{X}\right]+\sum_{j}\left(\hat{L}_{j}^{(S)} \hat{X} \hat{L}_{j}^{(S) \dagger}-\frac{1}{2}\left\{\hat{L}_{j}^{(S) \dagger} \hat{L}_{j}^{(S)}, \hat{X}\right\}\right),
\end{gathered}
$$

and

$$
\hat{\hat{F}} \hat{X}=-\frac{i}{\hbar}\left[\hat{H}_{S B}, \hat{X}\right]
$$

It is easy to show that $\hat{\hat{B}} \hat{\hat{S}}=\hat{\hat{S}} \hat{\hat{B}}$ and $e^{\hat{\hat{S}} t+\hat{\hat{B}} t}=e^{\hat{\hat{S}} t} e^{\hat{\hat{B}} t}=e^{\hat{\hat{B}} t} e^{\hat{\hat{S}} t}$.

Next, we will also use the Nakajima-Zwanzig projector superoperators [16, 17]. The defining action of the Nakajima-Zwanzig projector $\hat{\hat{P}}$ is written as

$$
\hat{\hat{P}} \hat{X}(t)=\hat{\rho}_{B}\left(t_{0}\right) \otimes \operatorname{Tr}_{B}\{\hat{X}(t)\}
$$

for any $\hat{X}(t)$ and any initial time $t_{0}$. With these projectors and algebraic manipulations, we obtain the general equation,

$$
\frac{d}{d t}[\hat{\hat{P}} \hat{\alpha}(t)]=\int_{0}^{t} d t^{\prime}\left[\hat{\hat{P}} \hat{\hat{G}}(t) \hat{\hat{G}}\left(t^{\prime}\right) \hat{\hat{P}} \hat{\alpha}(t)\right],
$$


where

$$
\hat{\alpha}(t)=e^{-\hat{\hat{S}} t-\hat{\hat{B}} t} \hat{\rho}_{S B}(t)
$$

and

$$
\hat{\hat{G}}(t)=e^{-\hat{\hat{S}} t-\hat{\hat{B}} t} \hat{\hat{F}} e^{\hat{\hat{S}} t+\hat{\hat{B}} t}
$$

Evidently, according to Eq. (17), once $\hat{\alpha}(t)$ is calculated, $\hat{\rho}_{S}(t)$ can be found by the action of $e^{\hat{\hat{S}} t}$ on the reduced $\hat{\alpha}(t)$, that is,

$$
\hat{\rho}_{S}(t)=e^{\hat{\hat{S}} t} \operatorname{Tr}_{B}\{\hat{\alpha}(t)\}
$$

As we explain in the Introduction, here we consider a two-state system interacting with a bath of harmonic oscillators, that emulates a phase-noisy environment. Thus, we take

$$
\begin{gathered}
\hat{H}_{S}=\hbar \omega_{0} \hat{\sigma}_{z}, \\
\hat{H}_{B}=\hbar \sum_{k} \omega_{k} \hat{b}_{k}^{\dagger} \hat{b}_{k},
\end{gathered}
$$

and phase-damping interaction [15], that is,

$$
\left\{\begin{array}{l}
\hat{S}_{k}=\hbar \hat{\sigma}_{z}, \\
\hat{B}_{k}=g_{k} \hat{b}_{k}^{\dagger}+g_{k}^{*} \hat{b}_{k} .
\end{array}\right.
$$

where $\omega_{0}$ and the $\omega_{k}$ are real constants, $\hat{b}_{k}$ and $\hat{b}_{k}^{\dagger}$ are the annihilation and creation bath operators, $g_{k}$ are complex coefficients. Here and next, we will use the Pauli matrices $\hat{\sigma}_{z}$ and $\hat{\sigma}_{x}$,

$$
\hat{\sigma}_{z}=\left(\begin{array}{cc}
1 & 0 \\
0 & -1
\end{array}\right), \hat{\sigma}_{x}=\left(\begin{array}{ll}
0 & 1 \\
1 & 0
\end{array}\right) .
$$

Hence, let us define the operator

$$
\hat{B} \equiv \sum_{k} \hat{B}_{k}=\sum_{k}\left(g_{k} \hat{b}_{k}^{\dagger}+g_{k}^{*} \hat{b}_{k}\right)
$$

Therefore, the interaction can be written in the simplified form:

$$
\hat{H}_{S B}=\hbar \hat{\sigma}_{z} \hat{B},
$$


that is,

$$
\hat{H}_{S B}=\hbar \sum_{k} \hat{\sigma}_{z}\left(g_{k} \hat{b}_{k}^{\dagger}+g_{k}^{*} \hat{b}_{k}\right)
$$

In the case of a finite temperature, we take the initial state of the environment as given by

$$
\hat{\rho}_{B}=\frac{1}{Z_{B}} \prod_{k} e^{-\hbar \beta \omega_{k} \hat{b}_{k}^{\dagger} \hat{b}_{k}}, Z_{B}=\prod_{l} \frac{1}{1-e^{-\hbar \beta \omega_{l}}} .
$$

\section{SOLUTION OF THE HYBRID MASTER EQUATION}

In this section, we solve Eq. (6) analytically in the cases where $\hat{L}^{(S)}=\lambda \hat{\sigma}_{z}$ at finite

temperature, and $\hat{L}^{(S)}=\lambda \hat{\sigma}_{x}$, under the assumptions that $\hat{H}_{S}=0$ and $T=0$. The quantity $\hat{\hat{P}} \hat{\alpha}(t)$ appears on both sides of Eq. (6) and we can simplify it:

$$
\hat{\hat{P}} \hat{\alpha}(t)=\hat{\hat{P}} e^{-\hat{\hat{S}} t-\hat{\hat{B}} t} \hat{\rho}_{S B}(t)=e^{-\hat{\hat{S}} t} \hat{\rho}_{S}(t) \operatorname{Tr}_{B}\left\{e^{-\hat{\hat{B}} t} \hat{\rho}_{B}\right\} \hat{\rho}_{B}=e^{-\hat{\hat{S}} t} \hat{\rho}_{S}(t) \hat{\rho}_{B}
$$

Now let us define the operator:

$$
\hat{R}(t) \equiv e^{-\hat{\hat{S}} t} \hat{\rho}_{S}(t)
$$

Hence,

$$
\hat{\hat{P}} \hat{\alpha}(t)=\hat{R}(t) \hat{\rho}_{B}
$$

Therefore, to recover the reduced density operator of the system, we apply $e^{\hat{\hat{S}} t}$ to $\hat{R}(t)$ :

$$
\hat{\rho}_{S}(t)=e^{\hat{\hat{S}} t} \hat{R}(t)
$$

An unusual aspect that should be clarified is the action of the superoperator exponentials, $e^{\hat{\hat{S}} t}$ and $e^{\hat{\hat{B}} t}$. Let us consider, initially, the time-independent operators $\hat{X}^{\prime}$ and $\hat{X}$, related by the operation $e^{\hat{\hat{B}} t}$ :

$$
\hat{X}^{\prime}=e^{\hat{\hat{B}} t} \hat{X}
$$

When we take the time derivative of $\hat{X}^{\prime}$ and use Eq. (14), we obtain 


$$
\frac{d}{d t} \hat{X}^{\prime}=\hat{\hat{B}} e^{\hat{\hat{B}} t} \hat{X}=\hat{\hat{B}} \hat{X}^{\prime}
$$

Now, if we consider the definition of the superoperator $\hat{\hat{B}}$, Eq. (3), we obtain the elementary Liouville-von Neumann equation, that is,

$$
\frac{d}{d t} \hat{X}^{\prime}=-\frac{i}{\hbar}\left[\hat{H}_{B}, \hat{X}^{\prime}\right]
$$

whose solution is easily determined for a time-independent $\hat{H}_{B}$, as in the case of (9):

$$
\hat{X}^{\prime}=e^{\hat{\hat{B}} t} \hat{X}=e^{-i \frac{\hat{H}_{B}}{\hbar} t} \hat{X} e^{i \frac{\hat{H}_{B}}{\hbar} t}
$$

\section{A. Expanding the integrand that appears in the hybrid master equation}

In view of Eq. (12), the integrand can be written as $\hat{\hat{P}} \hat{\hat{G}}(t) \hat{\hat{G}}\left(t^{\prime}\right) \hat{R}(t) \hat{\rho}_{B}$. From Eq. (8)), we obtain

$$
\begin{aligned}
\hat{\hat{G}}(t) \hat{\hat{G}}\left(t^{\prime}\right) \hat{R}(t) \hat{\rho}_{B} & =i e^{-\hat{\hat{S}} t} e^{-\hat{\hat{B}} t} \hat{\hat{F}}\left\{e^{\hat{\hat{S}}\left(t-t^{\prime}\right)}\left[\left(e^{\hat{\hat{S}} t^{\prime}} \hat{R}(t)\right) \hat{\sigma}_{z}\right]\right\} \\
& \times\left\{e^{\hat{\hat{B}}\left(t-t^{\prime}\right)}\left[\left(e^{\hat{\hat{B}} t^{\prime}} \hat{\rho}_{B}\right) \hat{B}\right]\right\} \\
& -i e^{-\hat{\hat{S}} t} e^{-\hat{\hat{B}} t} \hat{\hat{F}}\left\{e^{\hat{\hat{S}}\left(t-t^{\prime}\right)}\left[\hat{\sigma}_{z}\left(e^{\hat{\hat{S}} t^{\prime}} \hat{R}(t)\right)\right]\right\} \\
& \times\left\{e^{\hat{\hat{B}}\left(t-t^{\prime}\right)}\left[\hat{B}\left(e^{\hat{\hat{B}} t^{\prime}} \hat{\rho}_{B}\right)\right]\right\} .
\end{aligned}
$$


From Eqs. (44) and (5), we can rewrite Eq. (16) as

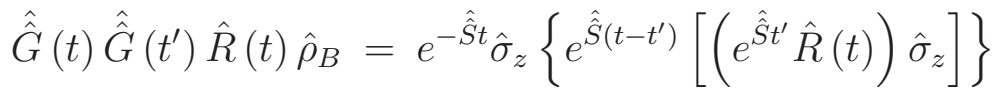

$$
\begin{aligned}
& \times \underbrace{\operatorname{Tr}_{B}\left\{e^{-\hat{\hat{B} t}} \hat{B}\left\{e^{\hat{\hat{B}}\left(t-t^{\prime}\right)}\left[\left(e^{\hat{\hat{B}} t^{\prime}} \hat{\rho}_{B}\right) \hat{B}\right]\right\}\right\}}_{(I)} \otimes \hat{\rho}_{B} \\
& \text { - } e^{-\hat{\hat{S} t}}\left\{e^{\hat{\hat{S}}\left(t-t^{\prime}\right)}\left[\left(e^{\hat{\hat{S}} t^{\prime}} \hat{R}(t)\right) \hat{\sigma}_{z}\right]\right\} \hat{\sigma}_{z} \\
& \times \underbrace{\operatorname{Tr}_{B}\left\{e^{-\hat{\hat{B}} t}\left\{e^{\hat{\hat{B}}\left(t-t^{\prime}\right)}\left[\left(e^{\hat{B} t^{\prime}} \hat{\rho}_{B}\right) \hat{B}\right]\right\} \hat{B}\right\}}_{(I I)} \otimes \hat{\rho}_{B} \\
& -e^{-\hat{S} t} \hat{\sigma}_{z}\left\{e^{\hat{\hat{S}}\left(t-t^{\prime}\right)}\left[\hat{\sigma}_{z}\left(e^{\hat{\hat{S}} t^{\prime}} \hat{R}(t)\right)\right]\right\} \\
& \times \underbrace{\operatorname{Tr}_{B}\left\{e^{-\hat{\hat{B}} t} \hat{B}\left\{e^{\hat{\hat{B}}\left(t-t^{\prime}\right)}\left[\hat{B}\left(e^{\hat{\hat{B}} t^{\prime}} \hat{\rho}_{B}\right)\right]\right\}\right\}}_{(I I I)} \otimes \hat{\rho}_{B} \\
& +e^{-\hat{\hat{S} t}}\left\{e^{\hat{\hat{S}}\left(t-t^{\prime}\right)}\left[\hat{\sigma}_{z}\left(e^{\hat{\hat{S}} t^{\prime}} \hat{R}(t)\right)\right]\right\} \hat{\sigma}_{z} \\
& \times \underbrace{\operatorname{Tr}_{B}\left\{e^{-\hat{\hat{B}} t}\left\{e^{\hat{\hat{B}}\left(t-t^{\prime}\right)}\left[\hat{B}\left(e^{\hat{\hat{B}} t^{\prime}} \hat{\rho}_{B}\right)\right]\right\} \hat{B}\right\}}_{(I V)} \otimes \hat{\rho}_{B} .
\end{aligned}
$$

It is interesting to note that, in Eq. (17), the actions of $S$ and $B$ are completely separated in each term appearing on the right-hand side. This proves extremely valuable in the calculations that follow.

\section{B. Tracing out the environmental degrees of freedom}

For the sake of convenience, let us analyze, firstly, the environment quantities appearing in Eq. (17). According to Appendix A, the partial trace over the environmental variables gives: 


$$
\begin{aligned}
\hat{\hat{P}} \hat{\hat{G}}(t) \hat{\hat{G}}\left(t^{\prime}\right) \hat{\hat{P}} \hat{\alpha}(t)= & \left\{e^{-\hat{\hat{S}} t} \hat{\sigma}_{z}\left\{e^{\hat{\hat{S}}\left(t-t^{\prime}\right)}\left[\left(e^{\hat{\hat{S}} t^{\prime}} \hat{R}(t)\right) \hat{\sigma}_{z}\right]\right\}\right. \\
& \left.-e^{-\hat{\hat{S}} t}\left\{e^{\hat{\hat{S}}\left(t-t^{\prime}\right)}\left[\left(e^{\hat{\hat{S}} t^{\prime}} \hat{R}(t)\right) \hat{\sigma}_{z}\right]\right\} \hat{\sigma}_{z}\right\} \otimes \hat{\rho}_{B} \\
\times & \sum_{l}\left|g_{l}\right|^{2}\left\{\operatorname{coth}\left(\frac{\hbar \beta \omega_{l}}{2}\right) \cos \left[\omega_{l}\left(t-t^{\prime}\right)\right]+i \sin \left[\omega_{l}\left(t-t^{\prime}\right)\right]\right\} \\
+ & \left\{e^{-\hat{\hat{S}} t}\left\{e^{\hat{\hat{S}}\left(t-t^{\prime}\right)}\left[\hat{\sigma}_{z}\left(e^{\hat{\hat{S}} t^{\prime}} \hat{R}(t)\right)\right]\right\} \hat{\sigma}_{z}\right. \\
& \left.-e^{-\hat{\hat{S}} t} \hat{\sigma}_{z}\left\{e^{\hat{\hat{S}}\left(t-t^{\prime}\right)}\left[\hat{\sigma}_{z}\left(e^{\hat{\hat{S}} t^{\prime}} \hat{R}(t)\right)\right]\right\}\right\} \otimes \hat{\rho}_{B} \\
\times & \sum_{l}\left|g_{l}\right|^{2}\left\{\operatorname{coth}\left(\frac{\hbar \beta \omega_{l}}{2}\right) \cos \left[\omega_{l}\left(t-t^{\prime}\right)\right]-i \sin \left[\omega_{l}\left(t-t^{\prime}\right)\right]\right\} .
\end{aligned}
$$

\section{Introducing a continuous density of states characterizing the environment}

In Eq. (18), if we adopt the general definition of the density of states as

$$
J(\omega)=\sum_{l}\left|g_{l}\right|^{2} \delta\left(\omega-\omega_{l}\right)
$$


then the sum over the index $l$ can be replaced by an integral over a continuum of frequencies:

$$
\begin{aligned}
& \hat{\hat{P}} \hat{\hat{G}}(t) \hat{\hat{G}}\left(t^{\prime}\right) \hat{\hat{P}} \hat{\alpha}(t)=\int_{0}^{\infty} d \omega J(\omega)\left\{\operatorname{coth}\left(\frac{\hbar \beta \omega}{2}\right) \cos \left[\omega\left(t-t^{\prime}\right)\right]+i \sin \left[\omega\left(t-t^{\prime}\right)\right]\right\} \otimes \hat{\rho}_{B} \\
& \times\{\underbrace{e^{-\hat{\hat{S}} t} \hat{\sigma}_{z}\left\{e^{\hat{\hat{S}}\left(t-t^{\prime}\right)}\left[\left(e^{\hat{\hat{S}} t^{\prime}} \hat{R}(t)\right) \hat{\sigma}_{z}\right]\right\}}_{(A)} \\
& -\underbrace{e^{-\hat{\hat{S}} t}\left\{e^{\hat{\hat{S}}\left(t-t^{\prime}\right)}\left[\left(e^{\hat{\hat{S}} t^{\prime}} \hat{R}(t)\right) \hat{\sigma}_{z}\right]\right\} \hat{\sigma}_{z}}_{(B)}\} \\
& +\int_{0}^{\infty} d \omega J(\omega)\left\{\operatorname{coth}\left(\frac{\hbar \beta \omega}{2}\right) \cos \left[\omega\left(t-t^{\prime}\right)\right]-i \sin \left[\omega\left(t-t^{\prime}\right)\right]\right\} \otimes \hat{\rho}_{B} \\
& \times\{\underbrace{e^{-\hat{\hat{S}} t}\left\{e^{\hat{\hat{S}}\left(t-t^{\prime}\right)}\left[\hat{\sigma}_{z}\left(e^{\hat{\hat{S}} t^{\prime}} \hat{R}(t)\right)\right]\right\} \hat{\sigma}_{z}}_{(C)} \\
& -\underbrace{e^{-\hat{\hat{S}} t} \hat{\sigma}_{z}\left\{e^{\hat{\hat{S}}\left(t-t^{\prime}\right)}\left[\hat{\sigma}_{z}\left(e^{\hat{S} t^{\prime}} \hat{R}(t)\right)\right]\right\}}_{(D)}\} .
\end{aligned}
$$

Here, to obtain an analytical solution, we choose the Ohmic density of states (19):

$$
J(\omega)=\eta \omega e^{-\frac{\omega}{\omega_{c}}}
$$

where $\eta, \omega_{c} \geqslant 0$ and $\eta$ is the constant that gives the intensity of the coupling between the system and its environment.

\section{Reduced density operator describing the system}

To obtain the action of the operator $e^{\hat{\hat{S}} t}$, it is necessary to solve Eq. (1) without the environment. Then, we take

$$
\hat{H}=\hat{H}_{S}=\hbar \omega_{0} \hat{\sigma}_{z}
$$

and, in the Lindbladian, $\hat{L}^{(S)}=\lambda \hat{\sigma}_{z}$ or $\hat{L}^{(S)}=\lambda \hat{\sigma}_{x}$. 
In the case of $\hat{L}^{(S)}=\lambda \hat{\sigma}_{z}$, the solution is simple and can be found in Ref. [14]:

$$
\left\{\begin{array}{l}
\rho_{11}^{(z)}(t)=\rho_{11}^{(z)}(0) \\
\rho_{12}^{(z)}(t)=\rho_{12}^{(z)}(0) e^{-2 \lambda^{2} t} e^{-i 2 \omega_{0} t}
\end{array}\right.
$$

where the upper index $(z)$ indicates that the solution is written in the eigenbasis of $\hat{\sigma}_{z}$.

For $\hat{L}^{(S)}=\lambda \hat{\sigma}_{x}$, the solution is more complicated:

$$
\left\{\begin{aligned}
\rho_{11}^{(z)}(t) & =\frac{1}{2}+\frac{2 \rho_{11}^{(z)}(0)-1}{2} e^{-2 \lambda^{2} t} \\
\rho_{12}^{(z)}(t) & =e^{-\lambda^{2} t}\left\{\rho_{12}^{(z)}(0) \cosh \left(\sqrt{\lambda^{4}-4 \omega_{0}^{2}} t\right)\right. \\
& -\rho_{12}^{(z)}(0) \frac{i 2 \omega_{0}}{\sqrt{\lambda^{4}-4 \omega_{0}^{2}}} \sinh \left(\sqrt{\lambda^{4}-4 \omega_{0}^{2}} t\right) \\
& \left.+\frac{\lambda^{2}}{\sqrt{\lambda^{4}-4 \omega_{0}^{2}}} \rho_{12}^{(z) *}(0) \sinh \left(\sqrt{\lambda^{4}-4 \omega_{0}^{2}} t\right)\right\} .
\end{aligned}\right.
$$

To analyze the result of a measurement it is natural to represent the density operator in the eigenbasis of the measuring apparatus. Accordingly, in the present case, we use the eigenstates of $\hat{\sigma}_{x}$, that is,

$$
\left\{\begin{array}{l}
|+\rangle_{x}=\frac{|+\rangle+|-\rangle}{\sqrt{2}} \\
|-\rangle_{x}=\frac{|+\rangle-|-\rangle}{\sqrt{2}}
\end{array}\right.
$$

The change of basis is performed with the eigenvectors matrix

$$
\hat{M}=\frac{1}{\sqrt{2}}\left(\begin{array}{cc}
1 & 1 \\
1 & -1
\end{array}\right)=\hat{M}^{-1}
$$


and the result is:

$$
\left\{\begin{aligned}
\rho_{11}^{(x)}(t) & =\frac{1}{2}+e^{-\lambda^{2} t}\left\{\cosh \left(\sqrt{\lambda^{4}-4 \omega_{0}^{2}} t\right) \operatorname{Re}\left\{\rho_{12}^{(z)}(t)\right\}\right. \\
& +\frac{2 \omega_{0}}{\sqrt{\lambda^{4}-4 \omega_{0}^{2}}} \sinh \left(\sqrt{\lambda^{4}-4 \omega_{0}^{2}} t\right) \operatorname{Im}\left\{\rho_{12}^{(z)}(t)\right\} \\
& \left.+\frac{\lambda^{2}}{\sqrt{\lambda^{4}-4 \omega_{0}^{2}}} \sinh \left(\sqrt{\lambda^{4}-4 \omega_{0}^{2}} t\right) \operatorname{Re}\left\{\rho_{12}^{(z)}(t)\right\}\right\}, \\
\rho_{12}^{(x)}(t) & =\frac{2 \rho_{11}^{(z)}(t)-1}{2} e^{-2 \lambda^{2} t}-i e^{-\lambda^{2} t}\left\{\cosh \left(\sqrt{\lambda^{4}-4 \omega_{0}^{2}} t\right) \operatorname{Im}\left\{\rho_{12}^{(z)}(t)\right\}\right. \\
& -\frac{2 \omega_{0}}{\sqrt{\lambda^{4}-4 \omega_{0}^{2}}} \sinh \left(\sqrt{\lambda^{4}-4 \omega_{0}^{2}} t\right) \operatorname{Re}\left\{\rho_{12}^{(z)}(t)\right\} \\
& \left.-\frac{\lambda^{2}}{\sqrt{\lambda^{4}-4 \omega_{0}^{2}}} \sinh \left(\sqrt{\lambda^{4}-4 \omega_{0}^{2}} t\right) \operatorname{Im}\left\{\rho_{12}^{(z)}(t)\right\}\right\} .
\end{aligned}\right.
$$

E. The case of $\hat{L}^{(S)}=\lambda \hat{\sigma}_{z}$

Here, we consider the evolution of the system in contact with a thermal reservoir at arbitrary temperature, i.e., we assume that the initial condition of the environment is given by Eq. (11).

Let us write

$$
\hat{R}(t)=\left(\begin{array}{ll}
R_{11} & R_{12} \\
R_{21} & R_{22}
\end{array}\right)
$$

where, for notational convenience, we take $R_{i j}=R_{i j}(t)$. Then, using Eq. (22), we obtain

$$
e^{\hat{\hat{S}} t^{\prime}} \hat{R}(t)=\left(\begin{array}{cc}
R_{11} & R_{12} e^{2 \lambda^{2} t^{\prime}} e^{-i 2 \omega_{0} t^{\prime}} \\
R_{21} e^{2 \lambda^{2} t^{\prime}} e^{i 2 \omega_{0} t^{\prime}} & R_{22}
\end{array}\right)
$$

Substituting this into Eq. (20) and manipulating the terms according to Appendix B gives

$$
\frac{d}{d t}\left(\begin{array}{ll}
R_{11} & R_{12} \\
R_{21} & R_{22}
\end{array}\right)=-4\left(\begin{array}{cc}
0 & R_{12} \\
R_{21} & 0
\end{array}\right) \int_{0}^{t} d t^{\prime} \int_{0}^{\infty} d \omega J(\omega) \cos \left[\omega\left(t-t^{\prime}\right)\right] \operatorname{coth}\left(\frac{\beta \hbar \omega}{2}\right) .
$$

\section{Obtaining the populations}

According to Eq. (27), the populations are independent of $J(\omega)$ and can be immediately evaluated, giving 


$$
\frac{d}{d t} R_{i i}=0 \Rightarrow R_{i i}(t)=R_{i i}(0)
$$

where $i=1,2$. Then, using the constraint that the trace of the density operator must equal unity, we obtain

$$
\left\{\begin{array}{l}
\rho_{11}(t)=\rho_{11}(0) \\
\rho_{22}(t)=1-\rho_{11}(0) .
\end{array}\right.
$$

\section{Obtaining the coherences}

From Eq. (27) it follows that the non-diagonal elements satisfy

$$
\frac{d}{d t} R_{i j}=-4 \eta R_{i j}(t) \int_{0}^{t} d t^{\prime} \int_{0}^{\infty} d \omega \omega e^{-\frac{\omega}{\omega_{c}}} \cos \left[\omega\left(t-t^{\prime}\right)\right] \operatorname{coth}\left(\frac{\beta \hbar \omega}{2}\right)
$$

where $i, j=1,2$ and $i \neq j$. From the procedure of Appendix C, we obtain

$$
\rho_{12}(t)=\rho_{12}(0)\left[\frac{\Gamma\left(\frac{1}{\omega_{c} \beta \hbar}+i \frac{t}{\beta \hbar}\right) \Gamma\left(\frac{1}{\omega_{c} \beta \hbar}-i \frac{t}{\beta \hbar}\right)}{\Gamma^{2}\left(\frac{1}{\omega_{c} \beta \hbar}\right)} \frac{\Gamma\left(\frac{1}{\omega_{c} \beta \hbar}+1+i \frac{t}{\beta \hbar}\right) \Gamma\left(\frac{1}{\omega_{c} \beta \hbar}+1-i \frac{t}{\beta \hbar}\right)}{\Gamma^{2}\left(\frac{1}{\omega_{c} \beta \hbar}+1\right)}\right]^{2 \eta} e^{-2 \lambda^{2} t} e^{i 2 \omega_{0} t},
$$

where $\Gamma$ denotes the gamma function. Simplifying Eq. (30), according to Appendix D, gives

$$
\rho_{12}(t)=\rho_{12}(0)\left\{\frac{1}{1+\left(\omega_{c} t\right)^{2}}\left[\prod_{n=1}^{\infty} \frac{1+n \omega_{c} \beta \hbar}{1+n \omega_{c} \beta \hbar+\omega_{c} t}\right]^{2}\right\}^{2 \eta} e^{-2 \lambda^{2} t} e^{i 2 \omega_{0} t} .
$$

The consistency of Eqs. (30) and (31) with the case without environment can be verified by noticing that, in the limit $\eta \rightarrow 0$ (i.e., in the absence of environment), they reduce to Eq. (22), as expected.

F. The case of $\hat{L}^{(S)}=\lambda \hat{\sigma}_{x}$

From Eq. (23) the action of $e^{\hat{\hat{S}} t}$ on an operator

$$
\hat{x}=\left(\begin{array}{ll}
x_{11} & x_{12} \\
x_{21} & x_{22}
\end{array}\right)
$$

gives 


$$
e^{\hat{\hat{S}} t} \hat{x}=\left(\begin{array}{ll}
s_{11} & s_{12} \\
s_{21} & s_{22}
\end{array}\right),
$$

where

$$
\begin{gathered}
\left\{\begin{array}{l}
s_{11}=\frac{x_{11}-x_{22}}{2} e^{-2 \lambda^{2} t}+\frac{x_{11}+x_{22}}{2}, \\
s_{22}=-\frac{x_{11}-x_{22}}{2} e^{-2 \lambda^{2} t}+\frac{x_{11}+x_{22}}{2},
\end{array}\right. \\
\left\{\begin{array}{l}
s_{12}=\frac{e^{-\lambda^{2} t}}{\Omega}\left[\Omega \cosh (\Omega t) x_{12}-i 2 \omega_{0} \sinh (\Omega t) x_{12}+\lambda^{2} \sinh (\Omega t) x_{21}\right], \\
s_{21}=\frac{e^{-\lambda^{2} t}}{\Omega}\left[\Omega \cosh (\Omega t) x_{21}+i 2 \omega_{0} \sinh (\Omega t) x_{21}+\lambda^{2} \sinh (\Omega t) x_{12}\right],
\end{array}\right.
\end{gathered}
$$

with

$$
\Omega \equiv \sqrt{\lambda^{4}-4 \omega_{0}^{2}}
$$

According to the procedure explained in Appendix E, using Eqs. (32) and (33) in the case of the operator

$$
\hat{R}(t)=\left(\begin{array}{ll}
r_{11}(t) & r_{12}(t) \\
r_{21}(t) & r_{22}(t)
\end{array}\right)
$$

results in the final differential equation:

$$
\begin{aligned}
\frac{d}{d t}\left[\begin{array}{ll}
r_{11}(t) & r_{12}(t) \\
r_{21}(t) & r_{22}(t)
\end{array}\right] & =-\frac{4}{\Omega^{3}} \int_{0}^{t} d t^{\prime} \int_{0}^{\infty} d \omega J(\omega) \cos \left[\omega\left(t-t^{\prime}\right)\right] \operatorname{coth}\left(\frac{\beta \hbar \omega}{2}\right) \\
& \times\left[\begin{array}{cc}
Q_{1}\left(t, t^{\prime}\right) r_{12}(t)+Q_{2}\left(t, t^{\prime}\right) r_{21}(t) \\
(35)
\end{array}\right]
\end{aligned}
$$

where

$$
\left\{\begin{aligned}
Q_{1}\left(t, t^{\prime}\right) \equiv & K_{1}(t)\left[K_{1}^{*}\left(t-t^{\prime}\right) K_{1}^{*}\left(t^{\prime}\right)-K_{2}\left(t-t^{\prime}\right) K_{2}\left(t^{\prime}\right)\right] \\
& +K_{2}(t)\left[K_{2}\left(t-t^{\prime}\right) K_{1}^{*}\left(t^{\prime}\right)-K_{1}\left(t-t^{\prime}\right) K_{2}\left(t^{\prime}\right)\right] \\
Q_{2}\left(t, t^{\prime}\right) \equiv & K_{1}(t)\left[K_{1}^{*}\left(t-t^{\prime}\right) K_{2}\left(t^{\prime}\right)-K_{2}\left(t-t^{\prime}\right) K_{1}\left(t^{\prime}\right)\right] \\
& +K_{2}(t)\left[K_{2}\left(t-t^{\prime}\right) K_{2}\left(t^{\prime}\right)-K_{1}\left(t-t^{\prime}\right) K_{1}\left(t^{\prime}\right)\right]
\end{aligned}\right.
$$


and

$$
\left\{\begin{array}{l}
K_{1}(t) \equiv \Omega \cosh (\Omega t)+i 2 \omega_{0} \sinh (\Omega t) \\
K_{2}(t) \equiv \lambda^{2} \sinh (\Omega t)
\end{array}\right.
$$

1. The populations represented in the eigenbasis of $\hat{\sigma}_{z}$

Analogously to the case of $\hat{L}^{(S)}=\lambda \hat{\sigma}_{z}$ and $T \neq 0$, the populations do not depend on $J(\omega)$. From Eq. (35) we obtain

$$
\frac{d}{d t} r_{i i}=0 \Rightarrow r_{i i}(t)=r_{i i}(0)
$$

where $i=1,2$. We notice that $r_{i i}(0)=\rho_{11}^{(z)}(0)$ and, using Eq. (13), we can write:

$$
\rho_{11}^{(z)}(t)=\frac{2 \rho_{11}^{(z)}(0)-1}{2} e^{-2 \lambda^{2} t}+\frac{1}{2}
$$

2. The coherences represented in the eigenbasis of $\hat{\sigma}_{z}$, for $T=0$ and $\omega_{0}=0$

Since $r_{12}(t)$ is the complex conjugate of $r_{21}(t)$, we only need to calculate one of them. Let us introduce the new variable $\tau=t-t^{\prime}$. Hence, it follows from Eq. (35) that

$$
\begin{aligned}
\frac{d}{d t} r_{12}(t) & =-4 \frac{\eta}{\Omega^{3}} \int_{0}^{t} d \tau \int_{0}^{\infty} d \omega \omega e^{-\frac{\omega^{\prime}}{\omega_{c}}} \cos (\omega \tau) \operatorname{coth}\left(\frac{\beta \hbar \omega}{2}\right) \\
& \times\left[Q_{1}(t, t-\tau) r_{12}(t)+Q_{2}(t, t-\tau) r_{21}(t)\right] .
\end{aligned}
$$

There is no analytic solution for this equation at a finite temperature. However, as detailed in Appendix F, we have found the following result for $T=0$ and $\omega_{0}=0$ :

$$
\begin{aligned}
\rho_{12}^{(z)}(t) & =\operatorname{Re}\left\{\rho_{12}^{(z)}(0)\right\} e^{-8 \eta \lambda^{2} g_{0} t} e^{4 \eta \lambda^{2}\left[A_{-}(t)-B_{-}(t)\right]} \\
& +i \operatorname{Im}\left\{\rho_{12}^{(z)}(0)\right\} e^{-2 \lambda^{2} t} e^{8 \eta \lambda^{2} g_{0} t} e^{-4 \eta \lambda^{2}\left[A_{+}(t)+B_{+}(t)\right]}
\end{aligned}
$$


where

$$
\begin{aligned}
& A_{+}(t) \equiv \int_{0}^{t} e^{2 \lambda^{2} t^{\prime}} g_{1}\left(t^{\prime}\right) d t^{\prime} \\
& =\frac{1}{4 \lambda^{2}} \operatorname{Re}\left\{e^{i 2 \frac{\lambda^{2}}{\omega_{c}}}\left[e^{4 \lambda^{2} t} \Gamma\left(0,2 \lambda^{2} t+i 2 \frac{\lambda^{2}}{\omega_{c}}\right)-e^{-i 4 \frac{\lambda^{2}}{\omega_{c}}} \Gamma\left(0,-2 \lambda^{2} t-i 2 \frac{\lambda^{2}}{\omega_{c}}\right)\right]\right\} \\
& +\frac{1}{2 \lambda^{2}} \operatorname{Re}\left\{e^{-i 2 \frac{\lambda^{2}}{\omega_{c}}}\left[i 2 \frac{\lambda^{2}}{\omega_{c}} \Gamma\left(0,-2 \lambda^{2} t-i 2 \frac{\lambda^{2}}{\omega_{c}}\right)+\Gamma\left(1,-2 \lambda^{2} t-i 2 \frac{\lambda^{2}}{\omega_{c}}\right)\right]\right\} \\
& +t \operatorname{Re}\left\{e^{-i 2 \frac{\lambda^{2}}{\omega_{c}}} \Gamma\left(0,-2 \lambda^{2} t-i 2 \frac{\lambda^{2}}{\omega_{c}}\right)\right\}+\operatorname{Re}\left\{e^{-i 2 \frac{\lambda^{2}}{\omega_{c}}} c_{1}+e^{i 2 \frac{\lambda^{2}}{\omega_{c}}} c_{3}\right\}, \\
& A_{-}(t) \equiv \int_{0}^{t} e^{-2 \lambda^{2} t^{\prime}} g_{1}\left(t^{\prime}\right) d t^{\prime} \\
& =-\frac{1}{4 \lambda^{2}} \operatorname{Re}\left\{e^{-i 2 \frac{\lambda^{2}}{\omega_{c}}}\left[e^{-4 \lambda^{2} t} \Gamma\left(0,-2 \lambda^{2} t-i 2 \frac{\lambda^{2}}{\omega_{c}}\right)-e^{i 4 \frac{\lambda^{2}}{\omega_{c}}} \Gamma\left(0,2 \lambda^{2} t+i 2 \frac{\lambda^{2}}{\omega_{c}}\right)\right]\right\} \\
& +\frac{1}{2 \lambda^{2}} \operatorname{Re}\left\{e^{i 2 \frac{\lambda^{2}}{\omega_{c}}}\left[i 2 \frac{\lambda^{2}}{\omega_{c}} \Gamma\left(0,2 \lambda^{2} t+i 2 \frac{\lambda^{2}}{\omega_{c}}\right)-\Gamma\left(1,2 \lambda^{2} t+i 2 \frac{\lambda^{2}}{\omega_{c}}\right)\right]\right\} \\
& +t \operatorname{Re}\left\{e^{i 2 \frac{\lambda^{2}}{\omega_{c}}} \Gamma\left(0,2 \lambda^{2} t+i 2 \frac{\lambda^{2}}{\omega_{c}}\right)\right\}-\operatorname{Re}\left\{e^{i 2 \frac{\lambda^{2}}{\omega_{c}}} c_{1}^{*}+e^{-i 2 \frac{\lambda^{2}}{\omega_{c}}} c_{3}^{*}\right\}, \\
& B_{+}(t) \equiv \int_{0}^{t} e^{2 \lambda^{2} t^{\prime}} g_{2}\left(t^{\prime}\right) d t^{\prime} \\
& =\frac{1}{4 \lambda^{2}} \operatorname{Re}\left\{e^{i 2 \frac{\lambda^{2}}{\omega_{c}}}\left[e^{4 \lambda^{2} t} \Gamma\left(-1,2 \lambda^{2} t+i 2 \frac{\lambda^{2}}{\omega_{c}}\right)+e^{-i 4 \frac{\lambda^{2}}{\omega_{c}}} \Gamma\left(-1,-2 \lambda^{2} t-i 2 \frac{\lambda^{2}}{\omega_{c}}\right)\right]\right\} \\
& -\frac{1}{2 \lambda^{2}} \operatorname{Re}\left\{e^{-i 2 \frac{\lambda^{2}}{\omega_{c}}}\left[i 2 \frac{\lambda^{2}}{\omega_{c}} \Gamma\left(-1,-2 \lambda^{2} t-i 2 \frac{\lambda^{2}}{\omega_{c}}\right)+\Gamma\left(0,-2 \lambda^{2} t-i 2 \frac{\lambda^{2}}{\omega_{c}}\right)\right]\right\} \\
& -t \operatorname{Re}\left\{e^{-i 2 \frac{\lambda^{2}}{\omega_{c}}} \Gamma\left(-1,-2 \lambda^{2} t-i 2 \frac{\lambda^{2}}{\omega_{c}}\right)\right\}-\operatorname{Re}\left\{e^{-i 2 \frac{\lambda^{2}}{\omega_{c}}} c_{2}-e^{i 2 \frac{\lambda^{2}}{\omega_{c}}} c_{4}\right\}, \\
& B_{-}(t) \equiv \int_{0}^{t} e^{-2 \lambda^{2} t^{\prime}} g_{2}\left(t^{\prime}\right) d t^{\prime} \\
& =\frac{1}{4 \lambda^{2}} \operatorname{Re}\left\{e^{-i 2 \frac{\lambda^{2}}{\omega_{c}}}\left[e^{-4 \lambda^{2} t} \Gamma\left(-1,-2 \lambda^{2} t-i 2 \frac{\lambda^{2}}{\omega_{c}}\right)+e^{i 4 \frac{\lambda^{2}}{\omega_{c}}} \Gamma\left(-1,2 \lambda^{2} t+i 2 \frac{\lambda^{2}}{\omega_{c}}\right)\right]\right\} \\
& +\frac{1}{2 \lambda^{2}} \operatorname{Re}\left\{e^{i 2 \frac{\lambda^{2}}{\omega_{c}}}\left[i 2 \frac{\lambda^{2}}{\omega_{c}} \Gamma\left(-1,2 \lambda^{2} t+i 2 \frac{\lambda^{2}}{\omega_{c}}\right)-\Gamma\left(0,2 \lambda^{2} t+i 2 \frac{\lambda^{2}}{\omega_{c}}\right)\right]\right\} \\
& +t \operatorname{Re}\left\{e^{i 2 \frac{\lambda^{2}}{\omega_{c}}} \Gamma\left(-1,2 \lambda^{2} t+i 2 \frac{\lambda^{2}}{\omega_{c}}\right)\right\}-\operatorname{Re}\left\{e^{i 2 \frac{\lambda^{2}}{\omega_{c}}} c_{2}^{*}-e^{-i 2 \frac{\lambda^{2}}{\omega_{c}}} c_{4}^{*}\right\},
\end{aligned}
$$

and 


$$
\left\{\begin{array}{l}
c_{1}=-\frac{1}{2 \lambda^{2}}\left[i \frac{2 \lambda^{2}}{\omega_{C}} \Gamma\left(0,-i \frac{2 \lambda^{2}}{\omega_{C}}\right)+\Gamma\left(1,-i \frac{2 \lambda^{2}}{\omega_{C}}\right)\right], \\
c_{2}=-\frac{1}{2 \lambda^{2}}\left[i \frac{2 \lambda^{2}}{\omega_{C}} \Gamma\left(-1,-i \frac{2 \lambda^{2}}{\omega_{C}}\right)+\Gamma\left(0,-i \frac{2 \lambda^{2}}{\omega_{C}}\right)\right], \\
c_{3}=-\frac{1}{4 \lambda^{2}}\left[\Gamma\left(0, i \frac{2 \lambda^{2}}{\omega_{C}}\right)-e^{-i \frac{4 \lambda^{2}}{\omega_{C}}} \Gamma\left(0,-i \frac{2 \lambda^{2}}{\omega_{C}}\right)\right], \\
c_{4}=-\frac{1}{4 \lambda^{2}}\left[\Gamma\left(-1, i \frac{2 \lambda^{2}}{\omega_{C}}\right)+e^{-i \frac{4 \lambda^{2}}{\omega_{C}}} \Gamma\left(-1,-i \frac{2 \lambda^{2}}{\omega_{C}}\right)\right] .
\end{array}\right.
$$

3. The density matrix elements represented in the eigenbasis of $\hat{\sigma}_{x}$, for $T=0$ and $\omega_{0}=0$

Let us change the previous result for the coherences to the eigenbasis of $\hat{\sigma}_{x}$. Using Eq. (25) with $\omega_{0}=0$ we obtain

$$
\left\{\begin{array}{l}
\hat{\rho}_{11}^{(x)}(t)=\frac{1}{2}+\operatorname{Re}\left\{\rho_{12}^{(z)}(0)\right\} e^{-8 \eta \lambda^{2} g_{0} t} e^{4 \eta \lambda^{2}\left[A_{-}(t)-B_{-}(t)\right]} \\
\hat{\rho}_{12}^{(x)}(t)=\frac{2 \rho_{11}^{(z)}(0)-1}{2} e^{-2 \lambda^{2} t}-i \operatorname{Im}\left\{\rho_{12}^{(z)}(0)\right\} e^{-2 \lambda^{2} t} e^{8 \eta \lambda^{2} g_{0} t} e^{-4 \eta \lambda^{2}\left[A_{+}(t)+B_{+}(t)\right]}
\end{array}\right.
$$

\section{NUMERICAL METHOD}

The approximate analytical results detailed above have been compared against the exact numerical solution of the Lindblad equation, Eq. (1). The algorithm we use derives from the superoperator-splitting method [25], which is adequate for numerically solving a first-order differential equation of the form:

$$
\frac{d}{d t} \hat{\rho}_{S B}(t)=\hat{\hat{A}} \hat{\rho}_{S B}(t)+\hat{\hat{B}} \hat{\rho}_{S B}(t)
$$

As long as the two superoperators $\hat{\hat{A}}$ and $\hat{\hat{B}}$ are time-independent, the solution for the differential equation is

$$
\hat{\rho}_{S B}(t)=e^{(\hat{\hat{A}+\hat{B}}) t} \hat{\rho}_{S B}(0) .
$$

In case this exponential superoperator cannot be analytically found, but the $e^{\hat{\hat{A}} t}$ and $e^{\hat{\hat{B}} t}$ can, we may use the approximation

$$
e^{(\hat{\hat{A}+\hat{B}}) \Delta t}=e^{\hat{\hat{A}} \Delta t} e^{\hat{\hat{B}} \Delta t}+O\left(\Delta t^{2}\right)
$$

to expand the solution in terms of a product of $N$ short time steps of length $\Delta t$. The alternate application of these two superoperators, $e^{\hat{\hat{A}} \Delta t}$ and $e^{\hat{\hat{B}} \Delta t}$, comprises the superoperator-splitting method. 
For a finite-time measurement, the superoperator $\hat{\hat{A}}$ represents the Liouvillian and $\hat{\hat{B}}$ represents the Lindbladian. The form of the exponential of the time-independent Liouvillian is straightforward, while $e^{\hat{\hat{B}} \Delta t}$ behaves in the way described by Eqs. (32) and (33). As the behavior of both populations in the $\hat{\sigma}_{z}$ eigenbasis can be exactly described by a simple analytical formula, only the coherences need to be calculated numerically. Therefore, all the relevant information about the density matrix can be represented by a pseudo-spinor containing both coherences, on which the alternate action of $e^{\hat{\hat{A}} \Delta t}$ and $e^{\hat{\hat{B}} \Delta t}$ will be equivalent to matrix products:

$$
\left(\begin{array}{l}
\hat{\rho}_{12}(N \Delta t) \\
\hat{\rho}_{21}(N \Delta t)
\end{array}\right)=e^{-\lambda^{2}(N \Delta t)}\left[\hat{\hat{K}}_{+1}(\Delta t) A_{+1}(\Delta t)+\hat{\hat{K}}_{-1}(\Delta t) A_{-1}(\Delta t)\right]^{N}\left(\begin{array}{c}
\hat{\rho}_{12}(0) \\
\hat{\rho}_{21}(0)
\end{array}\right),
$$

where

$$
\hat{\hat{K}}_{q}(\Delta t) \hat{X} \equiv e^{-i \sum_{k} \omega_{k}\left(\hat{b}_{k}+q g_{k} / \omega_{k}\right)^{\dagger}\left(\hat{b}_{k}+q g_{k} / \omega_{k}\right) \Delta t} \hat{X} e^{i \sum_{k} \omega_{k}\left(\hat{b}_{k}-q g_{k} / \omega_{k}\right)^{\dagger}\left(\hat{b}_{k}-q g_{k} / \omega_{k}\right) \Delta t}
$$

and we defined the square matrices $A_{ \pm}(\Delta t)$ as

$$
\begin{aligned}
& A_{+1}(\Delta t) \equiv\left(\begin{array}{cc}
b_{+1}(\Delta t) & b_{-1}(\Delta t) \\
0 & 0
\end{array}\right), \\
& A_{-1}(\Delta t) \equiv\left(\begin{array}{cc}
0 & 0 \\
b_{-1}(\Delta t) & b_{+1}(\Delta t)
\end{array}\right),
\end{aligned}
$$

with

$$
b_{q}(\Delta t) \equiv \frac{1}{2}\left(e^{\lambda^{2} \Delta t}+q e^{-\lambda^{2} \Delta t}\right) .
$$

The binomial in Eq. (40) may be expanded in $N$ two-term summations. Also, as we are not interested in the total density matrix, but in the reduced part referring to the two-state system, we may take the partial trace over the degrees of freedom of the environment, to find:

$$
\begin{aligned}
\left(\begin{array}{c}
\rho_{12}(N \Delta t) \\
\rho_{21}(N \Delta t)
\end{array}\right) & =e^{-\lambda^{2}(N \Delta t)} \sum_{q_{1} \in\{-1,1\}} \ldots \sum_{q_{N} \in\{-1,1\}} \prod_{n=1}^{N}\left[A_{q_{n}}(\Delta t)\right] \\
& \times \operatorname{Tr}_{B}\left\{\prod_{n=1}^{N}\left[\hat{\hat{K}}_{q_{n}}(\Delta t)\right]|0\rangle\langle 0|\right\}\left(\begin{array}{c}
\rho_{12}(0) \\
\rho_{21}(0)
\end{array}\right),
\end{aligned}
$$


where we are considering the system initially separable from the environment, which starts in the vacuum state $(T=0)$.

Employing the result for the trace given in Appendix G and the easily-verified matrix identity,

$$
A_{r}(\Delta t) A_{s}(\Delta t)=b_{r s}(\Delta t)\left(\sigma_{x}\right)^{(1-r s) / 2} A_{s}(\Delta t),
$$

we find the final form for the algorithm, which is comprised of a sum of $2^{N}$ terms, each containing $N^{2}(N-1)$ factors and a product of up to two matrices:

$$
\begin{aligned}
\left(\begin{array}{c}
\rho_{12}(N \Delta t) \\
\rho_{21}(N \Delta t)
\end{array}\right)= & e^{-\lambda^{2}(N \Delta t)} \sum_{q_{1} \in\{-1,1\}} \ldots \sum_{q_{N} \in\{-1,1\}} \\
& \prod_{m, n=1}^{N}\left\{1+\frac{2\left(\omega_{c} \Delta t\right)^{-2}+\left(1-2|m-n|^{2}\right)}{\left[\left(\omega_{c} \Delta t\right)^{-2}+|m-n|^{2}\right]^{2}}\right\}^{-\eta q_{m} q_{n}} \\
\times & \prod_{n=1}^{N-1}\left[b_{q_{n} q_{n+1}}(\Delta t)\right]\left(\sigma_{x}\right)^{\left(1-q_{1} q_{N}\right) / 2} A_{q_{N}}(\Delta t)\left(\begin{array}{c}
\rho_{12}(0) \\
\rho_{21}(0)
\end{array}\right),
\end{aligned}
$$

which can be implemented in any programming language capable of handling large floatingpoint numbers and of calculating powers and exponentials. The results are then translated into elements of the density matrix in the basis of eigenvectors of $\hat{\sigma}_{x}$ by a simple transformation, Eq. (24).

\section{RESULTS AND DISCUSSION}

The final analytical expression for the finite-time $\hat{\sigma}_{x}$ measurement given in Eq. (39) is expected to agree with the numerical solution Eq. (42) for weak coupling with the environment, in our case represented by the dimensionless constant $\eta$. This fact is confirmed in Fig. 1, which compares the time evolution of the population $\rho_{11}(t)$ using both methods for different values of the system-environment coupling constant. There it can be seen that the discrepancy between the two graphs grows with $\eta$.

Having confirmed the reliability of our results, we proceed to compare the noisy measurement described by our formalism with the case where the system is not being measured. As can be seen in Fig. 2, systems evolving only under the influence of the environment $\left(\lambda^{2}=0\right)$ 


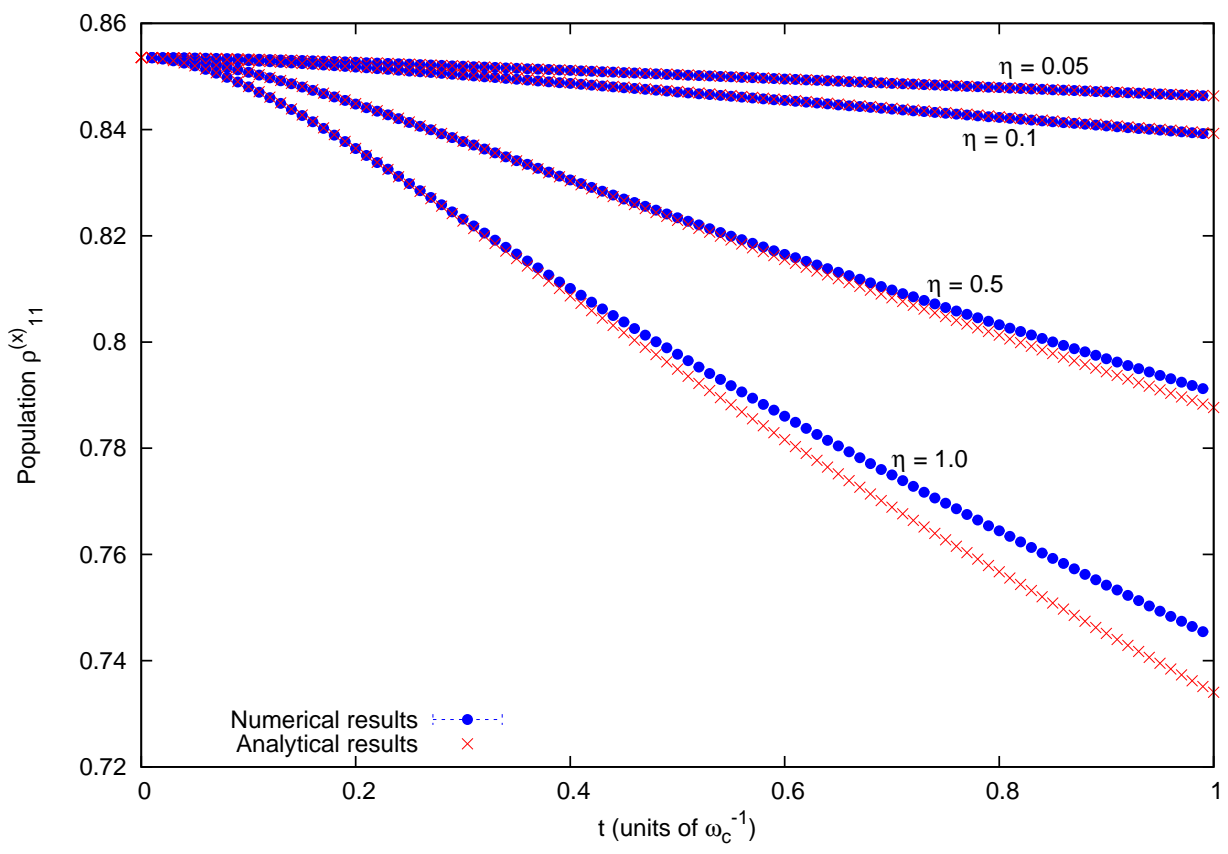

Figure 1: (Color online) Comparison between the time evolution of the population in the eigenbasis of $\hat{\sigma}_{x}, \rho_{11}^{(x)}(t)$, given by the analytical (crosses) and numerical (bullets) methods for $\lambda^{2}=4 \omega_{c}$ and different values of $\eta$. The system is initially in the pure state $\frac{1}{\sqrt{2}}\left(|1\rangle+e^{i \pi / 4}|2\rangle\right)$.

always have faster rates of change of their populations than those which are also interacting with the measurement apparatus $\left(\lambda^{2}>0\right)$. Moreover, the stronger the measurement, the slower the rate of change of the population. Therefore, a measurement of the observable $\hat{\sigma}_{x}$ helps to maintain the population closer to its original value.

The meaning of this phenomenon is straightforward: a difference between the initial value of the population (instant $t_{0}$ ) and its value at the end of the measurement (instant $t_{f}$ ) represents the probability that the measurement will give a wrong result. This is so because, if the initial value $\rho_{11}\left(t_{0}\right)$ and the final result $\rho_{11}\left(t_{f}\right)$ are different, then there will be a fraction of $\epsilon=\left|\rho_{11}\left(t_{f}\right)-\rho_{11}\left(t_{0}\right)\right|$ systems in the statistical ensemble that present final collapsed states different from those that would be obtained if we had measured them with an ideal instantaneous process at instant $t_{0}$. We shall henceforth refer to the difference $\epsilon$ as the error.

The fact that a finite-time measurement helps to preserve the initial value of the population of a system also shows that a naïve approach to a noisy measurement will overestimate the error. Given that a measurement ends at a time $t_{f}$ and that the system has been inter- 

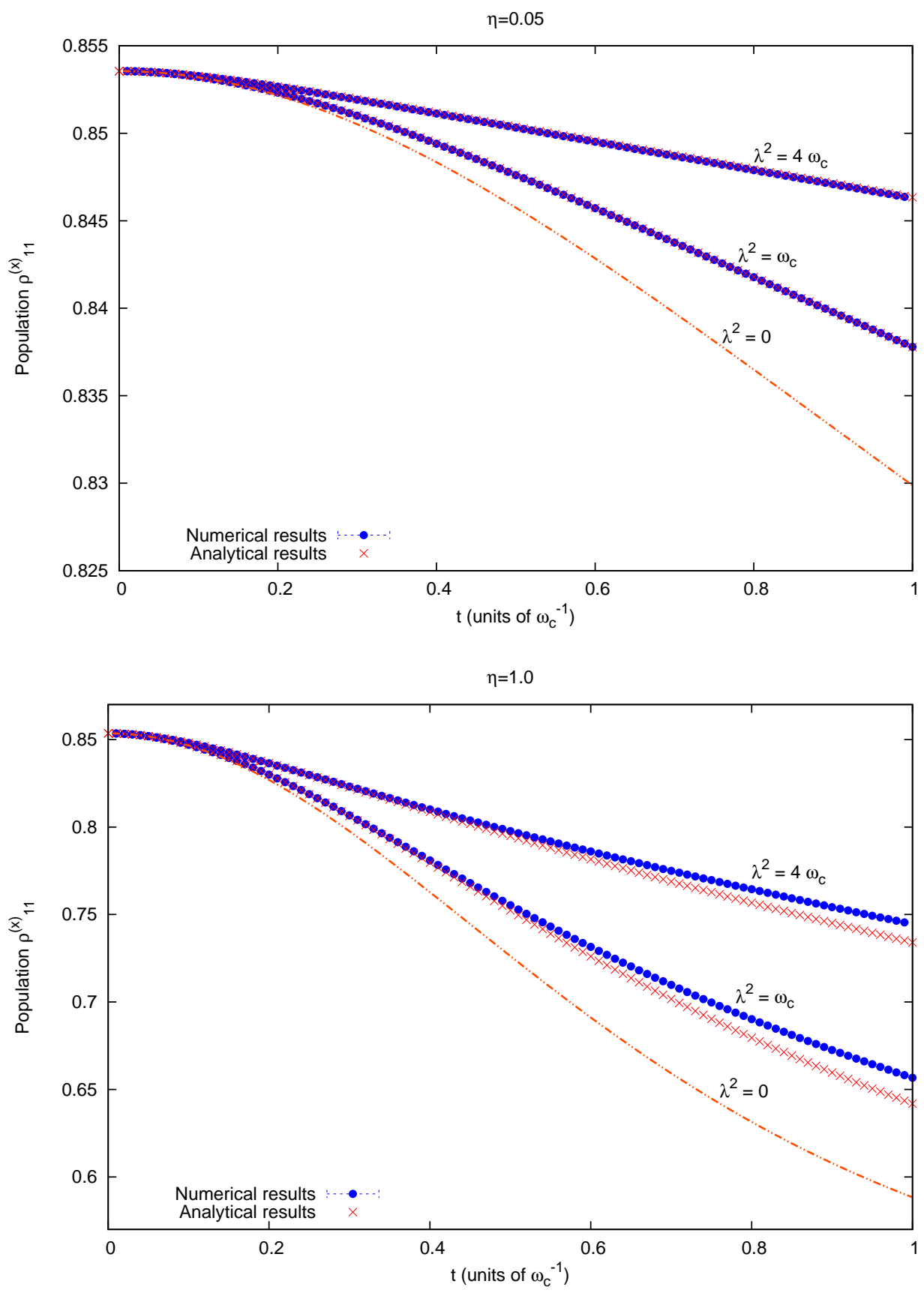

Figure 2: (Color online) Time evolution of the population in the eigenbasis of $\hat{\sigma}_{x}$ when there is an external environment causing phase noise (crosses for the analytical method and bullets for the numerical). The dashed line represents the evolution of a system that is only subject to the interaction with the environment $(\lambda=0)$. In all graphs, the evolution of the system that is not subject to measurement diverges faster from the initial value than those systems that are being measured. The initial conditions are the same applied to Fig. 1. 
acting with the environment since instant $t_{0}<t_{f}$, modeling the noisy measurement process as a period of interaction with the environment followed by an instantaneous measurement would lead to an error much larger than that obtained by considering that the system is measured continuously during a period $t_{f}-t_{0}$. Therefore, a description of the measurement as a continuous process is essential to have good estimates of the error.

Finally, it is important to notice that this protection against error depends on whether the observable measured commutes with the interaction Hamiltonian. In the measurement described above, the observable $\hat{\sigma}_{x}$ anti-commutes with the operator $\hat{\sigma}_{z}$ in the Hamiltonian. But when we measure an observable that does commute, the populations remain constant, and the only effect of the measuring apparatus is to strengthen the effects of the decoherence caused by the environment, as can be seen from Fig. 3 .

In short, when the observable measured is $\hat{\sigma}_{z}$, the measurement apparatus reinforces the effect of the environment, thus leading to faster decoherence. When the observable is $\hat{\sigma}_{x}$, the two effects compete against each other, leading to a smaller error than that expected for a system that is not subject to measurement.

\section{CONCLUSIONS AND PERSPECTIVES}

In this work, we have analyzed a two-state system subject to finite-time measurements that commute or anti-commute with the interaction Hamiltonian which couples the system with a phase-noisy environment. In the first case, we have shown that the complete analytical results for a finite-time measurement in any temperature - seen in Eqs. (28), (30) and (31) give only faster rates of decoherence as the strength of the measurement grows. In the latter case, we have arrived at an analytical expression for zero temperature in Eq. (39) which, together with the numerical method in Eq. (42), has made it possible to conclude that the initial state of the system under consideration is protected by the measurement. This result, a consequence of the fact that two simultaneous measurements of non-commuting observables interfere with each other, allows a deeper understanding of the measurement process by opening some interesting perspectives in the area of protection against errors and demonstrating that it is necessary to take into account that the measurement is a continuous process rather than an instantaneous collapse in order to have reliable estimates of the error.

Our approach of considering the system interacting with the environment, but the ap- 

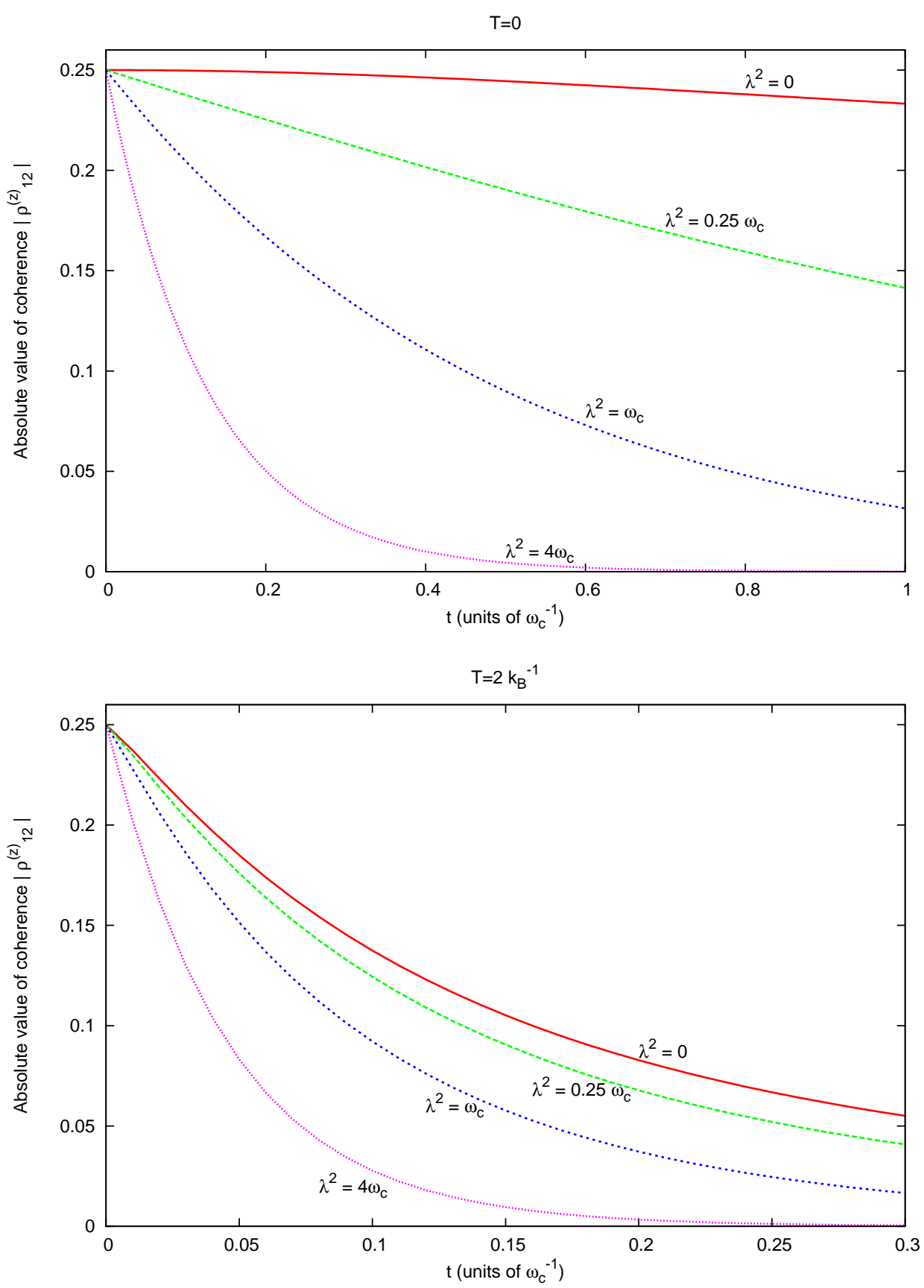

Figure 3: (Color online) Time evolution of the absolute value of the coherence in the eigenbasis of $\hat{\sigma}_{z}$ of a system suffering phase noise from an Ohmic environment $(\eta=0.05)$, while the observable $\hat{\sigma}_{z}$ is continuously measured. The curves were calculated using the analytical result of Eq. (31). The more the strength of measurement increases, the faster the coherence goes to zero. The initial conditions are the same applied in Fig. 1. 
paratus interacting only with the system, through our treatment of the Lindblad equation, is novel. However, we have treated the problem with a series of simplifications, namely: zero temperature for the initial state of the environment, Ohmic density of states, and the neglect of the system Hamiltonian when measuring an observable that does not commute with it. Subsequent studies may use our method in more general cases, even varying the number of states of the system considered. A first step in the direction of studying systems interacting with environments initially at temperature $T>0$ was given in Eq. (37), which shows the differential equations for the case of finite temperature that may serve for numerical resolutions that could reveal new effects.

The present formalism does not allow us to approach the possibility of the state measured being different from an eigenvalue of the system, as considered in some works on quantum theory of measurement [30]. However, it is still open the possibility of expanding our method for non-Markovian systems, time-dependent Nakajima-Zwanzig operators, and to analyze the quantum Zeno effect [33 35]. In particular, the quantum Zeno effect, viewed as a dynamical phenomenon [36], is closely connected with the fact that a finite-time measurement protects the initial state in the case of a measured observable that does not commute with the perturbing Hamiltonian. Roughly speaking, a finite-time measurement could be thought of as an approximation for a sequence of repeated instantaneous measurements, as in the bang-bang decoupling method [37], which has been generalized to arbitrarily fast and strong pulse sequences, requiring no symmetry. Therefore, we expect to be able to connect the findings of Ref. [37] with our present approach, clarifying how a general, finite-time measurement dynamically induces the quantum Zeno effect to protect the initial state being measured.

\section{Acknowledgments}

C. A. Brasil acknowledges support from Coordenação de Aperfeiçoamento de Pessoal de Nível Superior (CAPES), Brazil.

L. A. de Castro acknowledges support from Fundação de Amparo à Pesquisa do Estado de São Paulo (FAPESP), Brazil, project number 2009/12460-0.

R. d. J. Napolitano acknowledges support from Conselho Nacional de Desenvolvimento Científico e Tecnológico (CNPq), Brazil. 
Appendix A: Calculation of the environment degrees of freedom (Sec. iii-b)

Using Eq. (15), we can write:

(I) $\operatorname{Tr}_{B}\left\{e^{-\hat{\hat{B}} t} \hat{B}\left\{e^{\hat{\hat{B}}\left(t-t^{\prime}\right)}\left[\left(e^{\hat{\hat{B}} t^{\prime}} \hat{\rho}_{B}\right) \hat{B}\right]\right\}\right\}=\operatorname{Tr}_{B}\left\{e^{i \frac{\hat{H}_{B}}{\hbar} t} \hat{B} e^{-i \frac{\hat{H}_{B}}{\hbar} t} \hat{\rho}_{B} e^{i \frac{\hat{H}_{B}}{\hbar} t^{\prime}} \hat{B} e^{-i \frac{\hat{H}_{B}}{\hbar} t^{\prime}}\right\}$

$(I I) \operatorname{Tr}_{B}\left\{e^{-\hat{\hat{B}} t}\left\{e^{\hat{\hat{B}}\left(t-t^{\prime}\right)}\left[\left(e^{\hat{\hat{B}} t^{\prime}} \hat{\rho}_{B}\right) \hat{B}\right]\right\} \hat{B}\right\}=\operatorname{Tr}_{B}\left\{e^{i \frac{\hat{H}_{B}}{\hbar} t} \hat{B} e^{-i \frac{\hat{H}_{B}}{\hbar} t} \hat{\rho}_{B} e^{i \frac{\hat{H}_{B}}{\hbar} t^{\prime}} \hat{B} e^{-i \frac{\hat{H}_{B}}{\hbar} t^{\prime}}\right\}$

$(I I I) \operatorname{Tr}_{B}\left\{e^{-\hat{\hat{B}} t} \hat{B}\left\{e^{\hat{\hat{B}}\left(t-t^{\prime}\right)}\left[\hat{B}\left(e^{\hat{\hat{B}} t^{\prime}} \hat{\rho}_{B}\right)\right]\right\}\right\}=\operatorname{Tr}_{B}\left\{e^{i \frac{\hat{H}_{B}}{\hbar} t^{\prime}} \hat{B} e^{-i \frac{\hat{H}_{B}}{\hbar} t^{\prime}} \hat{\rho}_{B} e^{i \frac{\hat{H}_{B}}{\hbar} t} \hat{B} e^{-i \frac{\hat{H}_{B}}{\hbar} t}\right\}$

$(I V) \operatorname{Tr}_{B}\left\{e^{-\hat{\hat{B}} t}\left\{e^{\hat{\hat{B}}\left(t-t^{\prime}\right)}\left[\hat{B}\left(e^{\hat{\hat{B}} t^{\prime}} \hat{\rho}_{B}\right)\right]\right\} \hat{B}\right\}=\operatorname{Tr}_{B}\left\{e^{i \frac{\hat{H_{B}}}{\hbar} t^{\prime}} \hat{B} e^{-i \frac{\hat{H}_{B}}{\hbar} t^{\prime}} \hat{\rho}_{B} e^{i \frac{\hat{H}_{B}}{\hbar} t} \hat{B} e^{-i \frac{\hat{H}_{B}}{\hbar} t}\right\}$

Let us insert, then, Eqs. (43), (44), (45), and (46) into Eq. (17) and group the similar terms:

$$
\begin{aligned}
\hat{\hat{P}} \hat{\hat{G}}(t) \hat{\hat{G}}\left(t^{\prime}\right) \hat{\hat{P}} \hat{\alpha}(t) & =\left\{e^{-\hat{\hat{S}} t} \hat{\sigma}_{z}\left\{e^{\hat{\hat{S}}\left(t-t^{\prime}\right)}\left[\left(e^{\hat{\hat{S}} t^{\prime}} \hat{R}(t)\right) \hat{\sigma}_{z}\right]\right\}\right. \\
& \left.-e^{-\hat{\hat{S}} t}\left\{e^{\hat{\hat{S}}\left(t-t^{\prime}\right)}\left[\left(e^{\hat{\hat{S}} t^{\prime}} \hat{R}(t)\right) \hat{\sigma}_{z}\right]\right\} \hat{\sigma}_{z}\right\} \\
& \times \operatorname{Tr}_{B}\left\{e^{i \frac{\hat{H}_{B}}{\hbar} t} \hat{B} e^{-i \frac{\hat{H}_{B}}{\hbar} t} \hat{\rho}_{B} e^{i \frac{\hat{H}_{B}}{\hbar} t^{\prime}} \hat{B} e^{-i \frac{\hat{H}_{B}}{\hbar} t^{\prime}}\right\} \otimes \hat{\rho}_{B} \\
& +\left\{e^{-\hat{\hat{S}} t}\left\{e^{\hat{\hat{S}}\left(t-t^{\prime}\right)}\left[\hat{\sigma}_{z}\left(e^{\hat{\hat{S}} t^{\prime}} \hat{R}(t)\right)\right]\right\} \hat{\sigma}_{z}\right. \\
& \left.-e^{-\hat{\hat{S}} t} \hat{\sigma}_{z}\left\{e^{\hat{\hat{S}}\left(t-t^{\prime}\right)}\left[\hat{\sigma}_{z}\left(e^{\hat{\hat{S}} t^{\prime}} \hat{R}(t)\right)\right]\right\}\right\} \\
& \times \operatorname{Tr}_{B}\left\{e^{i \frac{\hat{H}_{B}}{\hbar} t^{\prime}} \hat{B} e^{-i \frac{\hat{H}_{B}}{\hbar} t^{\prime}} \hat{\rho}_{B} e^{i \frac{\hat{H}_{B}}{\hbar} t} \hat{B} e^{-i \frac{\hat{H}_{B}}{\hbar} t}\right\} \otimes \hat{\rho}_{B} .
\end{aligned}
$$


The two terms in Eq. (47) involving the trace over the environmental degrees of freedom have the same form. Then, it is sufficient to evaluate the first of them and perform the change

$$
\begin{cases}t & \rightarrow t^{\prime} \\ t^{\prime} & \rightarrow t\end{cases}
$$

to obtain the second. To take the partial trace, we use the Fock-state basis $\left|n_{1}\right\rangle \otimes\left|n_{2}\right\rangle \ldots \equiv$ $\prod_{m}\left|n_{m}\right\rangle$. Thus,

and we obtain [31]

$$
\operatorname{Tr}_{B}\{\} \rightarrow \sum_{n_{1}, n_{2}, \ldots}\left[\prod_{m}\left\langle n_{m}\right|\right]\left[\prod_{m}\left|n_{m}\right\rangle\right]
$$

$$
e^{i t \sum_{l} \omega_{l} \hat{b}_{l}^{\dagger} \hat{b}_{l}} \sum_{k}\left(g_{k}^{*} \hat{b}_{k}+g_{k} \hat{b}_{k}^{\dagger}\right) e^{-i t \sum_{l} \omega_{l} \hat{b}_{l}^{\dagger} \hat{b}_{l}}=\sum_{k}\left(g_{k}^{*} \hat{b}_{k} e^{-i \omega_{k} t}+g_{k} \hat{b}_{k}^{\dagger} e^{i \omega_{k} t}\right) .
$$

if we specify $\hat{H}_{B}$ and $\hat{B}$ as given by Eqs. (9) and (10). By Eq. (48), the trace over the environmental degrees of freedom becomes

$$
\begin{aligned}
\operatorname{Tr}_{B}\left\{e^{i \frac{\hat{H}_{B}}{\hbar} t} \hat{B} e^{-i \frac{\hat{H}_{B}}{\hbar} t} \hat{\rho}_{B} e^{i \frac{\hat{H}_{B}}{\hbar} t^{\prime}} \hat{B} e^{-i \frac{\hat{H}_{B}}{\hbar} t^{\prime}}\right\} & =\sum_{n_{1}, n_{2}, \ldots}\left[\prod_{m}\left\langle n_{m}\right|\right] \sum_{l}\left(g_{l}^{*} \hat{b}_{l} e^{-i \omega_{l} t}+g_{l} \hat{b}_{l}^{\dagger} e^{i \omega_{l} t}\right) \\
& \times \hat{\rho}_{B} \sum_{k}\left(g_{k}^{*} \hat{b}_{k} e^{-i \omega_{k} t^{\prime}}+g_{k} \hat{b}_{k}^{\dagger} e^{i \omega_{k} t^{\prime}}\right)\left[\prod_{m}\left|n_{m}\right\rangle\right] .
\end{aligned}
$$

With (11), we will obtain

$$
\begin{aligned}
\operatorname{Tr}_{B}\left\{e^{i \frac{\hat{H}_{B}}{\hbar} t} \hat{B} e^{-i \frac{\hat{H}_{B}}{\hbar} t} \hat{\rho}_{B} e^{i \frac{\hat{H}_{B}}{\hbar} t^{\prime}} \hat{B} e^{-i \frac{\hat{H}_{B}}{\hbar} t^{\prime}}\right\} & =\sum_{l}\left|g_{l}\right|^{2}\left[e^{i \omega_{l}\left(t-t^{\prime}\right)}+\frac{e^{i \omega_{l}\left(t-t^{\prime}\right)}}{e^{\hbar \beta \omega_{l}}-1}+\frac{e^{-i \omega_{l}\left(t-t^{\prime}\right)}}{e^{\hbar \beta \omega_{l}}-1}\right] \\
& =\sum_{l}\left|g_{l}\right|^{2}\left[e^{i \omega_{l}\left(t-t^{\prime}\right)}+\frac{2 \cos \left[\omega_{l}\left(t-t^{\prime}\right)\right]}{e^{\hbar \beta \omega_{l}}-1}\right],
\end{aligned}
$$

or, more simply,

$$
\begin{aligned}
\operatorname{Tr}_{B}\left\{e^{i \frac{\hat{H}_{B}}{\hbar} t} \hat{B} e^{-i \frac{\hat{H}_{B}}{\hbar} t} \hat{\rho}_{B} e^{i \frac{\hat{H}_{B}}{\hbar} t^{\prime}} \hat{B} e^{-i \frac{\hat{H}_{B}}{\hbar} t^{\prime}}\right\} & =\sum_{l}\left|g_{l}\right|^{2}\left\{\operatorname{coth}\left(\frac{\hbar \beta \omega_{l}}{2}\right) \cos \left[\omega_{l}\left(t-t^{\prime}\right)\right]\right. \\
& \left.+i \sin \left[\omega_{l}\left(t-t^{\prime}\right)\right]\right\}
\end{aligned}
$$


As explained above, the change of varibles

$$
\begin{cases}t & \rightarrow t^{\prime} \\ t^{\prime} & \rightarrow t\end{cases}
$$

in Eq. (49) gives us the other necessary term for Eq. (47), that is,

$$
\begin{aligned}
\operatorname{Tr}_{B}\left\{e^{i \frac{\hat{H}_{B}}{\hbar} t^{\prime}} \hat{B} e^{-i \frac{\hat{H}_{B}}{\hbar} t^{\prime}} \hat{\rho}_{B} e^{i \frac{\hat{H}_{B}}{\hbar} t} \hat{B} e^{-i \frac{\hat{H}_{B}}{\hbar} t}\right\} & =\sum_{l}\left|g_{l}\right|^{2}\left\{\operatorname{coth}\left(\frac{\hbar \beta \omega_{l}}{2}\right) \cos \left[\omega_{l}\left(t-t^{\prime}\right)\right]\right. \\
& \left.-i \sin \left[\omega_{l}\left(t-t^{\prime}\right)\right]\right\}
\end{aligned}
$$

The final result, Eq. (18), is achieved by inserting Eqs. (49) and (50) into Eq. (47).

Appendix B: the case of $\hat{L}^{(S)}=\lambda \hat{\sigma}_{z}$ (Sec. III-E)

Using Eq. (26) we can evaluate the terms $(A),(B),(C)$, and $(D)$ of Eq. (20). Therefore,

$$
\begin{aligned}
& (A) e^{-\hat{\hat{S}} t} \hat{\sigma}_{z}\left\{e^{\hat{\hat{S}}\left(t-t^{\prime}\right)}\left[\left(e^{\hat{\hat{S}} t^{\prime}} \hat{R}(t)\right) \hat{\sigma}_{z}\right]\right\}=\left(\begin{array}{cc}
R_{11} & -R_{12} \\
-R_{21} & R_{22}
\end{array}\right), \\
& \text { (B) } e^{-\hat{\hat{S}} t}\left\{e^{\hat{\hat{S}}\left(t-t^{\prime}\right)}\left[\left(e^{\hat{\hat{S}} t^{\prime}} \hat{R}(t)\right) \hat{\sigma}_{z}\right]\right\} \hat{\sigma}_{z}=\left(\begin{array}{cc}
R_{11} & R_{12} \\
R_{21} & R_{22}
\end{array}\right), \\
& \text { (C) } e^{-\hat{\hat{S}} t}\left\{e^{\hat{\hat{S}}\left(t-t^{\prime}\right)}\left[\hat{\sigma}_{z}\left(e^{\hat{\hat{S}} t^{\prime}} \hat{R}(t)\right)\right]\right\} \hat{\sigma}_{z}=\left(\begin{array}{cc}
R_{11} & -R_{12} \\
-R_{21} & R_{22}
\end{array}\right),
\end{aligned}
$$

and

$$
\text { (D) } e^{-\hat{\hat{S}} t} \hat{\sigma}_{z}\left\{e^{\hat{\hat{S}}\left(t-t^{\prime}\right)}\left[\hat{\sigma}_{z}\left(e^{\hat{\hat{S}} t^{\prime}} \hat{R}(t)\right)\right]\right\}=\left(\begin{array}{cc}
R_{11} & R_{12} \\
R_{21} & R_{22}
\end{array}\right) \text {. }
$$


With Eqs. (51), (52), (53), and (54), we can simplify Eq. (20):

$\hat{\hat{P}} \hat{\hat{G}}(t) \hat{\hat{G}}\left(t^{\prime}\right) \hat{\hat{P}} \hat{\alpha}(t)=-4 \int_{0}^{\infty} d \omega J(\omega)\left(\begin{array}{cc}0 & R_{12} \\ R_{21} & 0\end{array}\right) \cos \left[\omega\left(t-t^{\prime}\right)\right] \operatorname{coth}\left(\frac{\beta \hbar \omega}{2}\right) \otimes \hat{\rho}_{B}(55)$

Hence, we substitute Eq. (55) into the original Eq. (6) and obtain Eq. (27).

Appendix C: the coherence for the case of $\hat{L}^{(S)}=\lambda \hat{\sigma}_{z}$ (Sec. III-E.2)

In (29), for the sake of convenience, we perform the change of variable

$$
\begin{aligned}
\tau & =t-t^{\prime}, \\
d \tau & =-d t^{\prime}
\end{aligned}
$$

with

$$
\int_{0}^{t} d t^{\prime}=-\int_{t}^{0} d \tau=\int_{0}^{t} d \tau
$$

that gives

$$
\frac{d}{d t} R_{i j}=-4 \eta R_{i j}(t) \int_{0}^{t} d \tau \int_{0}^{\infty} d \omega \omega e^{-\frac{\omega}{\omega_{c}}} \cos (\omega \tau) \operatorname{coth}\left(\frac{\beta \hbar \omega}{2}\right) .
$$

The first step is to solve the time integral, that leads us to

$$
\frac{d}{d t} R_{i j}=-4 \eta R_{i j}(t) \int_{0}^{\infty} d \omega e^{-\frac{\omega}{\omega_{c}}} \sin (\omega t) \operatorname{coth}\left(\frac{\beta \hbar \omega}{2}\right)
$$

The frequency integral, evaluated with the help of Ref. [32], gives

$$
\begin{aligned}
\frac{d}{d t} R_{i j} & =i 2 \frac{\eta}{\beta \hbar} R_{i j}(t)\left[\psi\left(\frac{1}{\omega_{c} \beta \hbar}+i \frac{t}{\beta \hbar}\right)-\psi\left(\frac{1}{\omega_{c} \beta \hbar}-i \frac{t}{\beta \hbar}\right)\right. \\
& \left.+\psi\left(\frac{1}{\omega_{c} \beta \hbar}+1+i \frac{t}{\beta \hbar}\right)-\psi\left(\frac{1}{\omega_{c} \beta \hbar}+1-i \frac{t}{\beta \hbar}\right)\right]
\end{aligned}
$$

where

$$
\psi(z)=\frac{d}{d z} \ln [\Gamma(z)], \operatorname{Re}\{z\}>0
$$


The solution is, therefore,

$$
\begin{cases}R_{12}(t)= & R_{12}(0)\left[\frac{\Gamma\left(\frac{1}{\omega_{c} \beta \hbar}+i \frac{t}{\beta \hbar}\right) \Gamma\left(\frac{1}{\omega_{c} \beta \hbar}-i \frac{t}{\beta \hbar}\right)}{\Gamma^{2}\left(\frac{1}{\omega_{c} \beta \hbar}\right)} \frac{\Gamma\left(\frac{1}{\omega_{c} \beta \hbar}+1+i \frac{t}{\beta \hbar}\right) \Gamma\left(\frac{1}{\omega_{c} \beta \hbar}+1-i \frac{t}{\beta \hbar}\right)}{\Gamma^{2}\left(\frac{1}{\omega_{c} \beta \hbar}+1\right)}\right]^{2 \eta}, \\ R_{21}(t)= & R_{21}(0)\left[\frac{\Gamma\left(\frac{1}{\omega_{c} \beta \hbar}+i \frac{t}{\beta \hbar}\right) \Gamma\left(\frac{1}{\omega_{c} \beta \hbar}-i \frac{t}{\beta \hbar}\right)}{\Gamma^{2}\left(\frac{1}{\omega_{c} \beta \hbar}\right)} \frac{\Gamma\left(\frac{1}{\omega_{c} \beta \hbar}+1+i \frac{t}{\beta \hbar}\right) \Gamma\left(\frac{1}{\omega_{c} \beta \hbar}+1-i \frac{t}{\beta \hbar}\right)}{\Gamma^{2}\left(\frac{1}{\omega_{c} \beta \hbar}+1\right)}\right]^{2 \eta} .\end{cases}
$$

The coherences are obtained by performing the transformation of Eq. (13) to Eq. (56) . Since $\rho_{21}(t)=\rho_{12}^{*}(t)$, we consider only the element $\rho_{12}(t)$ and the result is Eq. (30).

Appendix D: Simplifying the expression of the coherence for the case of $\hat{L}^{(S)}=\lambda \hat{\sigma}_{z}$ (Sec. III-E.2)

Equation (30) can be simplified by using the properties of the gamma function [32]. With $\Gamma(x+1)=x \Gamma(x)$, we obtain

$$
\rho_{12}(t)=\rho_{12}(0)\left\{\frac{\Gamma^{2}\left(\frac{1}{\omega_{c} \beta \hbar}+i \frac{t}{\beta \hbar}\right) \Gamma^{2}\left(\frac{1}{\omega_{c} \beta \hbar}-i \frac{t}{\beta \hbar}\right)}{\Gamma^{4}\left(\frac{1}{\omega_{c} \beta \hbar}\right)}\left[1+\left(\omega_{c} t\right)^{2}\right]\right\}^{2 \eta} e^{-2 \lambda^{2} t} e^{i 2 \omega_{0} t} .
$$

Now, using the product representation

$$
\Gamma(z)=e^{-C z} \frac{1}{z} \prod_{k=1}^{\infty} \frac{e^{\frac{z}{k}}}{1+\frac{z}{k}}, \operatorname{Re}\{z\}>0
$$

where $C$ is the Euler constant,

$$
C=\lim _{s \rightarrow \infty}\left[\sum_{m=1}^{s} \frac{1}{m}-\ln (s)\right]=0.577215 \ldots
$$

we see that

$$
\frac{\Gamma\left(\frac{1}{\omega_{c} \beta \hbar}+i \frac{t}{\beta \hbar}\right) \Gamma\left(\frac{1}{\omega_{c} \beta \hbar}-i \frac{t}{\beta \hbar}\right)}{\Gamma^{2}\left(\frac{1}{\omega_{c} \beta \hbar}\right)}=\frac{1}{1+\left(\omega_{c} t\right)^{2}} \prod_{n=1}^{\infty} \frac{n \omega_{c} \beta \hbar+1}{1+n \omega_{c} \beta \hbar+\omega_{c} t}
$$

resulting, finally, in Eq. (31), which is suitable for numerical implementations.

Appendix E: the differential equations for the case of $\hat{L}^{(S)}=\lambda \hat{\sigma}_{x}$ (Sec. III-F)

Using the definitions (32) and (33) over (34), we have 


$$
e^{\hat{\hat{S}} t^{\prime}} \hat{R}(t)=e^{\hat{\hat{S}} t^{\prime}}\left(\begin{array}{ll}
r_{11} & r_{12} \\
r_{21} & r_{22}
\end{array}\right)=\left(\begin{array}{cc}
r_{11}^{(0)} & r_{12}^{(0)} \\
r_{21}^{(0)} & r_{22}^{(0)}
\end{array}\right)
$$

where

$$
\left\{\begin{array}{l}
r_{11}^{(0)}=\frac{r_{11}-r_{22}}{2} e^{-2 \lambda^{2} t^{\prime}}+\frac{r_{11}+r_{22}}{2} \\
r_{22}^{(0)}=-\frac{r_{11}-r_{22}}{2} e^{-2 \lambda^{2} t^{\prime}}+\frac{r_{11}+r_{22}}{2}
\end{array}\right.
$$

and

$$
\left\{\begin{array}{l}
r_{12}^{(0)}=\frac{e^{-\lambda^{2} t^{\prime}}}{\Omega}\left[\Omega \cosh \left(\Omega t^{\prime}\right) r_{12}-i 2 \omega_{0} \sinh \left(\Omega t^{\prime}\right) r_{12}+\lambda^{2} \sinh \left(\Omega t^{\prime}\right) r_{21}\right], \\
r_{21}^{(0)}=\frac{e^{-\lambda^{2} t^{\prime}}}{\Omega}\left[\Omega \cosh \left(\Omega t^{\prime}\right) r_{21}+i 2 \omega_{0} \sinh \left(\Omega t^{\prime}\right) r_{21}+\lambda^{2} \sinh \left(\Omega t^{\prime}\right) r_{12}\right] .
\end{array}\right.
$$

Now we can evaluate the terms between brackets on (20), that will furnish

$$
e^{\hat{\hat{S}}\left(t-t^{\prime}\right)}\left[\hat{\sigma}_{z}\left(e^{\hat{\hat{S}} t^{\prime}} \hat{R}(t)\right)\right]=\left(\begin{array}{cc}
r_{11}^{(1)} & r_{12}^{(1)} \\
r_{21}^{(1)} & r_{22}^{(1)}
\end{array}\right)
$$

and

$$
e^{\hat{\hat{S}}\left(t-t^{\prime}\right)}\left[\left(e^{\hat{\hat{S}} t^{\prime}} \hat{R}(t)\right) \hat{\sigma}_{z}\right]=\left(\begin{array}{cc}
r_{11}^{(2)} & r_{12}^{(2)} \\
r_{21}^{(2)} & r_{22}^{(2)}
\end{array}\right)
$$

where, using (57) and (58),

$$
\begin{aligned}
& \left\{\begin{array}{l}
r_{11}^{(1)}=\frac{r_{11}^{(0)}+r_{22}^{(0)}}{2} e^{-2 \lambda^{2}\left(t-t^{\prime}\right)}+\frac{r_{11}^{(0)}-r_{22}^{(0)}}{2}, \\
r_{22}^{(1)}=-\frac{r_{11}^{(0)}+r_{22}^{(0)}}{2} e^{-2 \lambda^{2}\left(t-t^{\prime}\right)}+\frac{r_{11}^{(0)}-r_{22}^{(0)}}{2}
\end{array}\right. \\
& \left\{\begin{array}{l}
r_{12}^{(1)}=\frac{e^{-\lambda^{2}\left(t-t^{\prime}\right)}}{\Omega}\left\{\Omega \cosh \left[\Omega\left(t-t^{\prime}\right)\right] r_{12}^{(0)}-i 2 \omega_{0} \sinh \left[\Omega\left(t-t^{\prime}\right)\right] r_{12}^{(0)}-\lambda^{2} \sinh \left[\Omega\left(t-t^{\prime}\right)\right] r_{21}^{(0)}\right\}, \\
r_{21}^{(1)}=-\frac{e^{-\lambda^{2}\left(t-t^{\prime}\right)}}{\Omega}\left\{\Omega \cosh \left[\Omega\left(t-t^{\prime}\right)\right] r_{21}^{(0)}+i 2 \omega_{0} \sinh \left[\Omega\left(t-t^{\prime}\right)\right] r_{21}^{(0)}-\lambda^{2} \sinh \left[\Omega\left(t-t^{\prime}\right)\right] r_{12}^{(0)}\right\},
\end{array}\right. \\
& \left\{\begin{array}{l}
r_{11}^{(2)}=\frac{r_{11}^{(0)}+r_{22}^{(0)}}{2} e^{-2 \lambda^{2}\left(t-t^{\prime}\right)}+\frac{r_{11}^{(0)}-r_{22}^{(0)}}{2} \\
r_{22}^{(2)}=-\frac{r_{11}^{(0)}+r_{22}^{(0)}}{2} e^{-2 \lambda^{2}\left(t-t^{\prime}\right)}+\frac{r_{11}^{(0)}-r_{22}^{(0)}}{2}
\end{array}\right.
\end{aligned}
$$


and

$$
\begin{aligned}
& \left\{\begin{array}{l}
r_{12}^{(2)}=-\frac{e^{-\lambda^{2}\left(t-t^{\prime}\right)}}{\Omega}\left\{\Omega \cosh \left[\Omega\left(t-t^{\prime}\right)\right] r_{12}^{(0)}-i 2 \omega_{0} \sinh \left[\Omega\left(t-t^{\prime}\right)\right] r_{12}^{(0)}-\lambda^{2} \sinh \left[\Omega\left(t-t^{\prime}\right)\right] r_{21}^{(0)}\right\}, \\
r_{21}^{(2)}=\frac{e^{-\lambda^{2}\left(t-t^{\prime}\right)}}{\Omega}\left\{\Omega \cosh \left[\Omega\left(t-t^{\prime}\right)\right] r_{21}^{(0)}+i 2 \omega_{0} \sinh \left[\Omega\left(t-t^{\prime}\right)\right] r_{21}^{(0)}-\lambda^{2} \sinh \left[\Omega\left(t-t^{\prime}\right)\right] r_{12}^{(0)}\right\},
\end{array}\right. \\
& \text { i.e., }\left\{\begin{array} { l } 
{ r _ { 1 1 } ^ { ( 1 ) } = r _ { 1 1 } ^ { ( 2 ) } } \\
{ r _ { 2 2 } ^ { ( 1 ) } = r _ { 2 2 } ^ { ( 2 ) } }
\end{array} \text { and } \left\{\begin{array}{l}
r_{12}^{(1)}=-r_{12}^{(2)} \\
r_{21}^{(1)}=-r_{21}^{(2)}
\end{array}\right.\right.
\end{aligned}
$$

The progressive calculus of $(A),(B),(C)$ and $(D)$, then, will furnish:

$$
\text { (A) } \quad e^{-\hat{\hat{S}} t} \hat{\sigma}_{z}\left\{e^{\hat{\hat{S}}\left(t-t^{\prime}\right)}\left[\left(e^{\hat{\hat{S}} t^{\prime}} \hat{R}(t)\right) \hat{\sigma}_{z}\right]\right\}=\left(\begin{array}{cc}
r_{11}^{(3)} & r_{12}^{(3)} \\
r_{21}^{(3)} & r_{22}^{(3)}
\end{array}\right)
$$

with

$$
\left\{\begin{array}{l}
r_{11}^{(3)}=\frac{r_{11}^{(2)}+r_{22}^{(2)}}{2} e^{2 \lambda^{2} t}+\frac{r_{11}^{(2)}-r_{22}^{(2)}}{2} \\
r_{22}^{(3)}=-\frac{r_{11}^{(2)}+r_{22}^{(2)}}{2} e^{2 \lambda^{2} t}+\frac{r_{11}^{(2)}-r_{22}^{(2)}}{2}
\end{array}\right.
$$

and

$$
\begin{aligned}
& \left\{\begin{array}{l}
r_{12}^{(3)}=\frac{e^{\lambda^{2} t}}{\Omega}\left[\Omega \cosh (\Omega t) r_{12}^{(2)}+i 2 \omega_{0} \sinh (\Omega t) r_{12}^{(2)}+\lambda^{2} \sinh (\Omega t) r_{21}^{(2)}\right] \\
r_{21}^{(3)}=-\frac{e^{\lambda^{2} t}}{\Omega}\left[\Omega \cosh (\Omega t) r_{21}^{(2)}-i 2 \omega_{0} \sinh (\Omega t) r_{21}^{(2)}+\lambda^{2} \sinh (\Omega t) r_{12}^{(2)}\right]
\end{array}\right. \\
& \text { (B) } e^{-\hat{\hat{S}} t}\left\{e^{\hat{\hat{S}}\left(t-t^{\prime}\right)}\left[\left(e^{\hat{\hat{S}} t^{\prime}} \hat{R}(t)\right) \hat{\sigma}_{z}\right]\right\} \hat{\sigma}_{z}=\left(\begin{array}{cc}
r_{11}^{(4)} & r_{12}^{(4)} \\
r_{21}^{(4)} & r_{22}^{(4)}
\end{array}\right)
\end{aligned}
$$

with

$$
\left\{\begin{array}{l}
r_{11}^{(4)}=\frac{r_{11}^{(2)}+r_{22}^{(2)}}{2} e^{2 \lambda^{2} t}+\frac{r_{11}^{(2)}-r_{22}^{(2)}}{2} \\
r_{22}^{(4)}=-\frac{r_{11}^{(2)}+r_{22}^{(2)}}{2} e^{2 \lambda^{2} t}+\frac{r_{11}^{(2)}-r_{22}^{(2)}}{2}
\end{array}\right.
$$

and

$$
\left\{\begin{array}{l}
r_{12}^{(4)}=-\frac{e^{\lambda^{2} t}}{\Omega}\left[\Omega \cosh (\Omega t) r_{12}^{(2)}+i 2 \omega_{0} \sinh (\Omega t) r_{12}^{(2)}+\lambda^{2} \sinh (\Omega t) r_{21}^{(2)}\right] \\
r_{21}^{(4)}=\frac{e^{\lambda^{2} t}}{\Omega}\left[\Omega \cosh (\Omega t) r_{21}^{(2)}-i 2 \omega_{0} \sinh (\Omega t) r_{21}^{(2)}+\lambda^{2} \sinh (\Omega t) r_{12}^{(2)}\right]
\end{array}\right.
$$


We can observe that $\left\{\begin{array}{l}r_{11}^{(3)}=r_{11}^{(4)} \\ r_{22}^{(3)}=r_{22}^{(4)}\end{array}\right.$ and $\left\{\begin{array}{l}r_{12}^{(3)}=-r_{12}^{(4)} \\ r_{21}^{(3)}=-r_{21}^{(4)}\end{array}\right.$
$(C) e^{-\hat{\hat{S}} t}\left\{e^{\hat{\hat{S}}\left(t-t^{\prime}\right)}\left[\hat{\sigma}_{z}\left(e^{\hat{\hat{S}} t^{\prime}} \hat{R}(t)\right)\right]\right\} \hat{\sigma}_{z}=\left(\begin{array}{cc}r_{11}^{(5)} & r_{12}^{(5)} \\ r_{21}^{(5)} & r_{22}^{(5)}\end{array}\right)$

with

$$
\left\{\begin{array}{l}
r_{11}^{(5)}=\frac{r_{11}^{(1)}+r_{22}^{(1)}}{2} e^{2 \lambda^{2} t}+\frac{r_{11}^{(1)}-r_{22}^{(1)}}{2} \\
r_{22}^{(5)}=-\frac{r_{11}^{(1)}+r_{22}^{(1)}}{2} e^{2 \lambda^{2} t}+\frac{r_{11}^{(1)}-r_{22}^{(1)}}{2}
\end{array}\right.
$$

and

$$
\begin{aligned}
& \left\{\begin{array}{l}
r_{12}^{(5)}=-\frac{e^{\lambda^{2} t}}{\Omega}\left[\Omega \cosh (\Omega t) r_{12}^{(1)}+i 2 \omega_{0} \sinh (\Omega t) r_{12}^{(1)}+\lambda^{2} \sinh (\Omega t) r_{21}^{(1)}\right] \\
r_{21}^{(5)}=\frac{e^{\lambda^{2} t}}{\Omega}\left[\Omega \cosh (\Omega t) r_{21}^{(1)}-i 2 \omega_{0} \sinh (\Omega t) r_{21}^{(1)}+\lambda^{2} \sinh (\Omega t) r_{12}^{(1)}\right]
\end{array}\right. \\
& \text { (D) } e^{-\hat{\hat{S}} t} \hat{\sigma}_{z}\left\{e^{\hat{\hat{S}}\left(t-t^{\prime}\right)}\left[\hat{\sigma}_{z}\left(e^{\hat{\hat{S}} t^{\prime}} \hat{R}(t)\right)\right]\right\}=\left(\begin{array}{cc}
r_{11}^{(6)} & r_{12}^{(6)} \\
r_{21}^{(6)} & r_{22}^{(6)}
\end{array}\right)
\end{aligned}
$$

with

$$
\left\{\begin{array}{l}
r_{11}^{(6)}=\frac{r_{11}^{(1)}+r_{22}^{(1)}}{2} e^{2 \lambda^{2} t}+\frac{r_{11}^{(1)}-r_{22}^{(1)}}{2} \\
r_{22}^{(6)}=-\frac{r_{11}^{(1)}+r_{22}^{(1)}}{2} e^{2 \lambda^{2} t}+\frac{r_{11}^{(1)}-r_{22}^{(1)}}{2}
\end{array}\right.
$$

and

$$
\left\{\begin{array}{l}
r_{12}^{(6)}=\frac{e^{\lambda^{2} t}}{\Omega}\left[\Omega \cosh (\Omega t) r_{12}^{(1)}+i 2 \omega_{0} \sinh (\Omega t) r_{12}^{(1)}+\lambda^{2} \sinh (\Omega t) r_{21}^{(1)}\right] \\
r_{21}^{(6)}=-\frac{e^{\lambda^{2} t}}{\Omega}\left[\Omega \cosh (\Omega t) r_{21}^{(1)}-i 2 \omega_{0} \sinh (\Omega t) r_{21}^{(1)}+\lambda^{2} \sinh (\Omega t) r_{12}^{(1)}\right]
\end{array}\right.
$$

Again, we have another similarity, $\left\{\begin{array}{l}r_{11}^{(5)}=r_{11}^{(6)} \\ r_{22}^{(5)}=r_{22}^{(6)}\end{array}\right.$ and $\left\{\begin{array}{l}r_{12}^{(5)}=-r_{12}^{(6)} \\ r_{21}^{(5)}=-r_{21}^{(6)}\end{array}\right.$.

By using (59), (60), (61) and (62), with the similarity relations, we have then the equation (35). 
Appendix F: the coherences in the case of $\hat{L}^{(S)}=\lambda \hat{\sigma}_{x}$ represented in the eigenbasis of $\hat{\sigma}_{z}$, for $T=0$ and $\omega_{0}=0$ (Sec. III-F.2)

For $T=0$ and $\omega_{0}=0$, the equation for the coherences becomes

$$
\begin{aligned}
\frac{d}{d t} r_{12}(t) & =4 \eta\left[\sinh \left(\lambda^{2} t\right) I_{2}(t)+\cosh \left(\lambda^{2} t\right) I_{3}(t)\right] r_{21}(t) \\
& -4 \eta\left[\cosh \left(\lambda^{2} t\right) I_{2}(t)+\sinh \left(\lambda^{2} t\right) I_{3}(t)\right] r_{12}(t),
\end{aligned}
$$

with

$$
\begin{gathered}
I_{2}(t)=2 \lambda^{2} \sinh \left(\lambda^{2} t\right) g_{0}+\lambda^{2} \sinh \left(\lambda^{2} t\right) g_{1}(t)+\lambda^{2} \cosh \left(\lambda^{2} t\right) g_{2}(t), \\
I_{3}(t)=-2 \lambda^{2} \cosh \left(\lambda^{2} t\right) g_{0}+\lambda^{2} \cosh \left(\lambda^{2} t\right) g_{1}(t)+\lambda^{2} \sinh \left(\lambda^{2} t\right) g_{2}(t),
\end{gathered}
$$

and

$$
\begin{gathered}
g_{0}=\operatorname{Re}\left\{\exp \left(i \frac{2 \lambda^{2}}{\omega_{C}}\right) \Gamma\left(0, i \frac{2 \lambda^{2}}{\omega_{C}}\right)\right\}, \\
g_{1}(t)=\operatorname{Re}\left\{\exp \left(2 \lambda^{2} t+i \frac{2 \lambda^{2}}{\omega_{C}}\right) \Gamma\left(0,2 \lambda^{2} t+i \frac{2 \lambda^{2}}{\omega_{C}}\right)\right. \\
\left.+\exp \left[-\left(2 \lambda^{2} t+i \frac{2 \lambda^{2}}{\omega_{C}}\right)\right] \Gamma\left[0,-\left(2 \lambda^{2} t+i \frac{2 \lambda^{2}}{\omega_{C}}\right)\right]\right\},
\end{gathered}
$$

and

$$
\begin{aligned}
g_{2}(t) & =\operatorname{Re}\left\{\exp \left[\left(2 \lambda^{2} t+i \frac{2 \lambda^{2}}{\omega_{C}}\right)\right] \Gamma\left[-1,\left(2 \lambda^{2} t+i \frac{2 \lambda^{2}}{\omega_{C}}\right)\right]\right. \\
& \left.-\exp \left[-\left(2 \lambda^{2} t+i \frac{2 \lambda^{2}}{\omega_{C}}\right)\right] \Gamma\left[-1,-\left(2 \lambda^{2} t+i \frac{2 \lambda^{2}}{\omega_{C}}\right)\right]\right\} .
\end{aligned}
$$

Let us write the complex conjugate of Eq. (63):

$$
\begin{aligned}
\frac{d}{d t} r_{21}(t) & =4 \eta\left[\sinh \left(\lambda^{2} t\right) I_{2}(t)+\cosh \left(\lambda^{2} t\right) I_{3}(t)\right] r_{12}(t) \\
& -4 \eta\left[\cosh \left(\lambda^{2} t\right) I_{2}(t)+\sinh \left(\lambda^{2} t\right) I_{3}(t)\right] r_{21}(t)
\end{aligned}
$$

Adding Eqs. (63) and (66) and subtracting Eq. (66) from (63), we obtain the decoupled system of equations: 


$$
\begin{cases}\frac{d}{d t} \operatorname{Re}\left\{r_{12}(t)\right\}= & 4 \eta\left[\sinh \left(\lambda^{2} t\right)-\cosh \left(\lambda^{2} t\right)\right]\left[I_{2}(t)-I_{3}(t)\right] \operatorname{Re}\left\{r_{12}(t)\right\}, \\ \frac{d}{d t} \operatorname{Im}\left\{r_{12}(t)\right\}= & -4 \eta\left[\sinh \left(\lambda^{2} t\right)+\cosh \left(\lambda^{2} t\right)\right]\left[I_{2}(t)+I_{3}(t)\right] \operatorname{Im}\left\{r_{12}(t)\right\} .\end{cases}
$$

Simplifying Eqs. (64) and (65) gives

$$
\begin{cases}\frac{d}{d t} \operatorname{Re}\left\{r_{12}(t)\right\}= & -4 \eta \lambda^{2}\left[2 g_{0}-e^{-2 \lambda^{2} t} g_{1}(t)+e^{-2 \lambda^{2} t} g_{2}(t)\right] \operatorname{Re}\left\{r_{12}(t)\right\}, \\ \frac{d}{d t} \operatorname{Im}\left\{r_{12}(t)\right\}= & 4 \eta \lambda^{2}\left[2 g_{0}-e^{2 \lambda^{2} t} g_{1}(t)-e^{2 \lambda^{2} t} g_{2}(t)\right] \operatorname{Im}\left\{r_{12}(t)\right\},\end{cases}
$$

whose solutions, in terms of the initial instant $t_{0}=0$, are given by

$$
\left\{\begin{aligned}
\operatorname{Re}\left\{r_{12}(t)\right\}= & \operatorname{Re}\left\{\rho_{12}^{(z)}(0)\right\} e^{-8 \eta \lambda^{2} g_{0} t} \exp \left\{4 \eta \lambda^{2} \int_{0}^{t} e^{-2 \lambda^{2} t^{\prime}} g_{1}\left(t^{\prime}\right) d t^{\prime}\right\} \\
& \times \exp \left\{-4 \eta \lambda^{2} \int_{0}^{t} e^{-2 \lambda^{2} t^{\prime}} g_{2}\left(t^{\prime}\right) d t^{\prime}\right\}, \\
\operatorname{Im}\left\{r_{12}(t)\right\}= & \operatorname{Im}\left\{\rho_{12}^{(z)}(0)\right\} e^{8 \eta \lambda^{2} g_{0} t} \exp \left\{-4 \eta \lambda^{2} \int_{0}^{t} e^{2 \lambda^{2} t^{\prime}} g_{1}\left(t^{\prime}\right) d t^{\prime}\right\} \\
& \times \exp \left\{-4 \eta \lambda^{2} \int_{0}^{t} e^{2 \lambda^{2} t^{\prime}} g_{2}\left(t^{\prime}\right) d t^{\prime}\right\} .
\end{aligned}\right.
$$

Calculating the integrals [32] results in Eq. (38).

\section{Appendix G: Calculation of the trace in Eq. (41)}

A convenient method for calculating the partial trace in Eq. (41) involves coherent states. For any sequence of $q_{1}, \ldots, q_{N}$, this partial trace takes the form of a matrix element of the vacuum:

$$
\begin{aligned}
\operatorname{Tr}_{B}\left\{\prod_{n=1}^{N}\left[\hat{\hat{K}}_{q_{n}}(\Delta t)\right]|0\rangle\langle 0|\right\} & =\prod_{k}\left\langle\left. 0\right|_{k} \prod_{n=0}^{N-1}\left[e^{i \omega_{k}\left(\hat{b}_{k}-q_{N-n} g_{k} / \omega_{k}\right)^{\dagger}\left(\hat{b}_{k}-q_{N-n} g_{k} / \omega_{k}\right) \Delta t}\right]\right. \\
& \times \prod_{n=1}^{N}\left[e^{-i \omega_{k}\left(\hat{b}_{k}+q_{n} g_{k} / \omega_{k}\right)^{\dagger}\left(\hat{b}_{k}+q_{n} g_{k} / \omega_{k}\right) \Delta t}\right]|0\rangle_{k} .
\end{aligned}
$$

The exponential operators present in this matrix element are best represented by displacement operators $\hat{D}_{k}\left(g_{k} / \omega_{k}\right)$ from quantum optics, which allow us to rewrite the partial trace as: 


$$
\begin{aligned}
\operatorname{Tr}_{B}\left\{\prod_{n=1}^{N}\left[\hat{\hat{K}}_{q_{n}}(\Delta t)\right]|0\rangle\langle 0|\right\} & =\prod_{k}\left\langle\left. 0\right|_{k} \prod_{n=0}^{N-1}\left[\hat{D}\left(q_{N-n} \frac{g_{k}}{\omega_{k}}\right) e^{i \omega_{k} \hat{b}_{k}^{\dagger} \hat{b}_{k} \Delta t} \hat{D}^{\dagger}\left(q_{N-n} \frac{g_{k}}{\omega_{k}}\right)\right]\right. \\
& \times \prod_{n=1}^{N}\left[\hat{D}^{\dagger}\left(q_{n} \frac{g_{k}}{\omega_{k}}\right) e^{-i \omega_{k} \hat{b}_{k}^{\dagger} \hat{b}_{k} \Delta t} \hat{D}\left(q_{n} \frac{g_{k}}{\omega_{k}}\right)\right]|0\rangle_{k} .
\end{aligned}
$$

Any sequence of these operators applied to a coherent state $\left|\alpha_{k}\right\rangle$ yields:

$$
\left[\hat{D}^{\dagger}\left(q_{n} \frac{g_{k}}{\omega_{k}}\right) e^{-i \omega_{k} \hat{b}_{k}^{\dagger} \hat{b}_{k} \Delta t} \hat{D}\left(q_{n} \frac{g_{k}}{\omega_{k}}\right)\right]\left|\alpha_{k}\right\rangle=\left|e^{-i \omega_{k} \Delta t} \alpha_{k}+\left(e^{-i \omega_{k} \Delta t}-1\right) q_{n} \frac{g_{k}}{\omega_{k}}\right\rangle
$$

where we have discarded the complex phase factors due to the displacement operators. Repeating the procedure $N$ times, we find that the partial trace is simply the inner product of coherent states:

$$
\prod_{k}\left\langle\sum_{n=1}^{N} e^{-i n \omega_{k} \Delta t}\left(e^{i \omega_{k} \Delta t}-1\right) q_{N-n+1} \frac{g_{k}}{\omega_{k}} \mid \sum_{n=1}^{N} e^{-i n \omega_{k} \Delta t}\left(1-e^{i \omega_{k} \Delta t}\right) q_{N-n+1} \frac{g_{k}}{\omega_{k}}\right\rangle .
$$

Such an inner product results in the exponential:

$$
\begin{aligned}
\operatorname{Tr}_{B}\left\{\prod_{n=1}^{N}\left[\hat{\hat{K}}_{q_{n}}(\Delta t)\right]|0\rangle\langle 0|\right\} & =\exp \left\{-8 \sum_{m=1}^{N} \sum_{n=1}^{N} q_{m} q_{n} \int_{0}^{\infty} d \omega \frac{J(\omega)}{\omega^{2}} \sin ^{2}\left(\frac{\omega \Delta t}{2}\right)\right. \\
& \times \cos [(m-n) \omega \Delta t]\}
\end{aligned}
$$

where we have taken the limit to a continuous spectrum of frequencies, applying the definition of the spectral-density function given in Eq. (19).

For Ohmic spectral densities, Eq. (21), the partial trace becomes the following exponential of an integral:

$$
\begin{aligned}
\operatorname{Tr}_{B}\left\{\prod_{n=1}^{N}\left[\hat{\hat{K}}_{q_{n}}(\Delta t)\right]|0\rangle\langle 0|\right\} & =\prod_{m=1}^{N} \prod_{n=1}^{N} \exp \left\{-8 \eta q_{m} q_{n} \int_{0}^{\infty} d \omega \frac{e^{-\omega / \omega_{c}}}{\omega} \sin ^{2}\left(\frac{\omega \Delta t}{2}\right)\right. \\
& \times \cos [(m-n) \omega \Delta t]\} .
\end{aligned}
$$

The remaining integral in $d \omega$ may be solved by first noticing that $\int_{0}^{\Delta t} d \tau \sin (\omega \tau)=$ $\frac{2}{\omega} \sin ^{2}\left(\frac{\omega \Delta t}{2}\right)$. This leads to a double integral that is easily solved as: 


$$
\begin{aligned}
4 \int_{0}^{\Delta t} d \tau \int_{0}^{\infty} d \omega e^{-\omega / \omega_{c}} \sin (\omega \tau) \cos [(m-n) \omega \Delta t] & =\ln \left\{1+\frac{2\left(\omega_{c} \Delta t\right)^{2}}{\left[1+(m-n)^{2}\left(\omega_{c} \Delta t\right)^{2}\right]^{2}}\right. \\
& \left.+\frac{\left[1-2(m-n)^{2}\right]\left(\omega_{c} \Delta t\right)^{4}}{\left[1+(m-n)^{2}\left(\omega_{c} \Delta t\right)^{2}\right]^{2}}\right\},
\end{aligned}
$$

which gives the following result for the trace:

$$
\operatorname{Tr}_{B}\left\{\prod_{n=1}^{N}\left[\hat{\hat{K}}_{q_{n}}(\Delta t)\right]|0\rangle\langle 0|\right\}=\prod_{m=1}^{N} \prod_{n=1}^{N}\left\{1+\frac{2\left(\omega_{c} \Delta t\right)^{-2}+\left[1-2(m-n)^{2}\right]}{\left[\left(\omega_{c} \Delta t\right)^{-2}+(m-n)^{2}\right]^{2}}\right\}^{-\eta q_{m} q_{n}}
$$

[1] C. Cohen-Tannoudji, B. Diu and F. Laloë, Quantum mechanics (Wiley, New York, 1977).

[2] J. A. Wheeler and W. H. Zurek, Quantum theory and measurement (Princeton University Press, Princeton, 1983).

[3] J. von Neumann, Mathematical foundations of quantum mechanics (Princeton University Press, Princeton, 1955).

[4] P. A. M. Dirac, The principles of quantum mechanics (Clarendon Press, Oxford, 1958).

[5] A. Bassi and G. Ghirardi, Phys. Rep. 379, 257 (2003).

[6] B. L. van der Waerden, Sources of quantum mechanics (North-Holland, Amsterdam, 1967).

[7] G. Ludwig, Wave mechanics (Pergamon Press, Oxford, 1968).

[8] P. A. M. Dirac, Proc. R. Soc. Lond. A 112, 661 (1926).

[9] A. Peres, Phys. Rev. A 61, 022116 (2000).

[10] S. L. Adler, Phys. Lett. A 265, 58 (2000).

[11] G. Lindblad, Commun. Math. Phys. 48, 119 (1976).

[12] E. B. Davies, Quantum theory of open systems (Academic Press, London, 1976).

[13] R. Alicki and K. Lendi, Quantum dynamical semigroups and applications (Springer-Verlag, Berlin, 2007).

[14] I. Percival, Quantum state diffusion (Cambridge University Press, Cambridge, 1998).

[15] H. P. Breuer and F. Petruccione, The theory of open quantum systems (Oxford University Press, Oxford, 2002).

[16] S. Nakajima, Progr. Theor. Phys. 20, 948 (1958). 
[17] R. Zwanzig, J. Chem. Phys. 33, 1338 (1960).

[18] C. A. Brasil and R. d. J. Napolitano, e-print arXiv:1102.3667v2.

[19] J. Fischer and H. P. Breuer, Phys. Rev. A 76, 052119 (2007).

[20] H. P. Breuer, Phys. Rev. A, 75, 022103 (2007).

[21] J. Seke, J. Phys. A: Math. Gen., 23, L61 (1990).

[22] A. Smirne and B. Vacchini, Phys. Rev. A 82, 022110 (2010).

[23] W. T. Strunz, L. Diosi and N. Gisin, Phys. Rev. Lett. 82, 1801 (1999).

[24] K. Dietz, J. Phys. A: Math. Gen., 36, L45 (2003).

[25] W. H. Press, S. A. Teukolsky, W. T. Vetterling and B. P. Flannery, Numerical Recipes - The Art of Scientific Computing (Cambridge University Press, New York, 2007).

[26] C. R. Willis and R. H. Picard, Phys. Rev. A 9, 1343 (1974).

[27] R. H. Picard and C. R. Willis, Phys. Rev. A 16, 1625 (1977).

[28] S. Jang, J. Cao and R. J. Silbey, J. Chem. Phys. 116, 2705 (2002).

[29] S. Jang, J. Cao and R. J. Silbey, J. Chem. Phys. 117, 10428 (2002).

[30] A. Peres and W. K. Wootters, Phys. Rev. D 32, 1968 (1985).

[31] R. Wilcox, J. Math. Phys. 8, 962 (1967).

[32] I.S. Gradshteyn and I.M. Ryzhik, Table of Integrals, Series, and Products 7th Edition (ElsevierAcademic Press, Burlington, 2007).

[33] A. Peres, Am. J. Phys. 48, 931 (1980).

[34] B. Misra and E. C. G. Sudarshan, J. Math. Phys. 18, 756 (1977).

[35] C. B. Chiu, E. C. G. Sudarshan and B. Misra, Phys. Rev. D 16, 520 (1977).

[36] P. Facchi and S. Pascazio, Phys. Rev. Lett. 89, 080401 (2002).

[37] P. Facchi, D. A. Lidar and S. Pascazio, Phys. Rev. A 69, 032314 (2004). 